\title{
The Schrödinger Equation for Quantum Fields with Nonlinear Nonlocal Scattering*
}

\author{
JAMES GLIMM \\ Department of Mathematics \\ Massachusetts Institute of Technology, \\ Matematisk Institut, Aarhus Universitet \\ Received February 18, 1966
}

\begin{abstract}
This paper considers perturbations $H=H_{0}+\varepsilon V$ of the Hamiltonian operator $H_{0}$ of a free scalar Boson field. $V$ is a polynomial in the annihilation creation operators. Terms of any order are allowed in $V$, but point interactions, such as $\int: \theta(x)^{4}: d x$, are not considered. Unnormalized solutions for the Schrödinger equation are found. For $\varepsilon \rightarrow 0$, these solutions have a partial asymptotic expansion in powers of $\varepsilon$. The set of all possible pertubation terms $V$ forms a Lie algebra. General properties of this Lie algebra are investigated.
\end{abstract}

\section{$\S 1$. Introduction}

We consider Hamiltonian operators of the form

$$
H=H_{0}+V
$$

where $H_{0}$ is the Hamiltonian for a free field and $V$ is a polynomial in the creation annihilation operators $A^{ \pm}$. By this we mean that $V$ is a finite sum of monomials $V_{l m}$ of the form

$$
V_{l m}=\int A^{+}\left(k_{1}\right) \ldots A^{+}\left(k_{l}\right) v_{l m}\left(k, k^{\prime}\right) A^{-}\left(k_{1}^{\prime}\right) \ldots A^{-}\left(k_{m}^{\prime}\right) d k d k^{\prime} .
$$

We require the kernel $v_{l m}$ to be smooth, for example to be in a Schwartz space $\mathscr{S}$. This paper is partly directed toward studying the Lie algebra formed by such $H$, and it is partly directed toward solving the Schrödinger equation

$$
i \frac{\partial}{\partial t} \Psi=H \Psi
$$

We solve (1.3) for quite general $V$ of the above form. (See Theorems 7.3 and 9.1.) We find in $\S 7$ a preliminary operator $T$ which intertwines $H$ and $H_{0}$,

Then

$$
H T=T H_{0} .
$$

$$
T \exp \left(-i t H_{0}\right) \Phi(0)=\Psi(t)
$$

* This work was supported in part by the National Science Foundation, NSF GP-4364.

Commun. math. Phys., Vol. 2 
is a solution of (1.3) with Cauchy data $\Psi(0)=T \Phi(0)$. We have not been able to identify the formal expression for $T^{-1}$ with an operator on any reasonable function space, and so we cannot specify "arbitrary" Cauchy data in (1.3). More serious defects in this $T$ are the following: (a) It seems that the associated scattering is trivial. (b) In perturbation theory (where $V$ is replaced by $\varepsilon V$ ), $T$ contains powers of $\varepsilon^{-1}$ as well as powers of $\varepsilon$. In fact, $T$ is a sum of terms $T_{l m}=T_{l m}(\varepsilon)$ such as (1.2) and each

$$
T_{l m}(\varepsilon)=\quad \sum_{j \geqq J(l, m) \varepsilon^{j} T_{l m}^{(j)}}
$$

has a pole of order $-J(l, m)$ depending on $l$ and $m$.

Combining this type of $T$ with familiar arguments from perturbation theory, we find in $\S 9$ new $T$ 's which have the property that $T_{l m}(\varepsilon)$ is analytic in $\varepsilon$ if $l \leqq N$. Here $N$ is a finite number which can be chosen in advance to be as large as desired. In order to do this we must make a finite renormalization of $H$. The resulting scattering appears to be nontrivial, and could probably be computed, using methods from [2]. This operator $T$ leads to solutions $\Psi=\Psi(t, \varepsilon)$ of the renormalized Schrödinger equation for which

$$
\psi_{0}(t, \varepsilon), \ldots, \psi_{N}(t, \varepsilon)
$$

depend analytically on $\varepsilon$. Here $\psi_{j}$ is the $j$ particle component of $\Psi$. The solution $\Psi$ will presumably not be normalized, and will exist as an element of a space larger than the Fock Hilbert space. These results should be compared to FrIEDRICHS' ideas [2]. In his terminology we have considered the case of a totally smooth interaction.

In $\S 2$ and $\S 3$ we realize our operators (1.1) as bounded operators on Frechet spaces, cf. [5]. In $\S 3$ we prove that the Lie algebra of $V$ 's in (1.1) has a trivial ideal theory. The only ideal in this Lie algebra is the one dimensional ideal,

$$
z=\left\{V: V=V_{00}\right\}
$$

which is its center. The significance for us of this result is primarily negative. Nontrivial ideals would simplify the search for our intertwinting operator $T$. We will later find $T$ as a product, $T=T_{1} T_{2}$ where in $T_{1}$ (respectively $T_{2}$ ) the creation (respectively annihilation) operators dominate. Thus

$$
\begin{aligned}
& \left(T_{1}\right)_{l m}=0 \text { if } l<m \\
& \left(T_{2}\right)_{l m}=0 \text { if } l>m .
\end{aligned}
$$

The operators of the form

$$
V=\sum_{l>m} V_{l m}
$$

form a subalgebra which in a generalized sense is nilpotent. In this algebra there are many ideals and the system of equations for the $\left(T_{i}\right)_{l m}$ can be solved successively. 
The algebra of all the $V$ 's is an algebra which, in a crude sense, is similar to the reductive Lie algebra $\mathfrak{g l}(n, C)$ of $n \times n$ complex matrices; the $T_{1}$ and $T_{2}$ correspond to the triangular matrices.

Since the Lie algebras we consider are infinite dimensional, it is not clear which topologies should be placed on them. In $\S 4$ we have some results which show that one natural choice for a topology does not seem to be suitable. In $\S 5$ we choose a better topology. For a $V$ of the form (1.7) the exponential map converges to an element of the Lie algebra. Thus we can identify the corresponding infinite dimensional Lie group (Theorem 5.6). This group acts by inner automorphisms on the full Lie algebra, and one of the group elements, namely $T_{1}$, puts $H$ in "triangular form". These considerations lead to our first $T$ in $\S 7$. The results of $\S 2-7$ do not depend very strongly on the class of function spaces we have considered. We could replace spaces of type $\mathscr{S}$ by spaces of rapidly decreasing continuous functions.

Sections 8 and 9 are devoted to the process of removing the poles from $T=T(\varepsilon)$, as mentioned above.

\section{$\S 2$. The operators $V$ and their domains}

Let $k_{i}$ be a vector in Euclidean three space. (We never refer to the components of $k_{i}$.) Let $\mu$ be a positive number and define

$$
\begin{aligned}
\omega\left(k_{i}\right) & =\left(\left|k_{i}\right|^{2}+\mu^{2}\right)^{1 / 2}, \\
H_{0} & =\int A^{+}(k) \omega(k) A^{-}(k) d k .
\end{aligned}
$$

Here $A^{+}$and $A^{-}$are the standard creation and annihilation operators for a scalar Boson field. This choice of statistics does not appear to be essential.

Let $\mathfrak{S}$ be the set of sequences

$$
\Phi=\left\{\varphi_{0}, \varphi_{1}, \ldots\right\}
$$

where $\varphi_{0}$ is a complex number and $\varphi_{n}$, for $n \geqq 1$, is a symmetric function of the variables $k_{1}, \ldots, k_{n}$ and where

$$
\varphi_{n} \in \mathscr{S} \text {. }
$$

We give $\mathfrak{D}$ the product topology. Then a net $\Phi^{(n)}$ converges if there is convergence $\varphi_{j}^{(n)} \rightarrow \varphi_{j}^{(\infty)}$ in each term. Let $\mathfrak{S}_{0}$ be the subset of $\mathfrak{D}$ consisting of those sequences $\Phi$ for which $\varphi_{n}=0$ for all sufficiently large $n$. Let

$$
\mathfrak{S}^{n}=\left\{\Phi: \varphi_{j}=0 \text { if } j>n\right\} .
$$

Then $\mathfrak{D}^{n}$ is a finite direct sum of spaces of type $\mathscr{S}$, and this defines a topology in $\mathfrak{S}^{n}$. Also

$$
\begin{aligned}
& \mathfrak{D}_{0}=\cup_{n} \mathfrak{D}^{n} \\
& \mathfrak{D}^{n} \subset \mathfrak{D}^{n+1} .
\end{aligned}
$$


We give $\mathscr{D}_{0}$ the inductive limit topology. A linear functional $L$ or a linear transformation $T$ defined on $\mathfrak{D}_{0}$ is continuous if and only if the restriction

or

$$
L \mid \mathfrak{D}^{n}
$$

$$
T \mid \mathfrak{S}^{n}
$$

is continuous for each $n$.

Let $\mathfrak{u}$ be set of all formal sums

$$
V=\Sigma V_{l m}
$$

where $V_{l m}$ is given by (1.2), $v_{l m}$ is in $\mathscr{S}$ and $v_{l m}$ is symmetric in the variables

and in the variables

$$
k_{1}, \ldots, k_{l}=k
$$

$$
k_{1}^{\prime}, \ldots, k_{m}^{\prime}=k^{\prime} \text {. }
$$

We give $\mathfrak{u}$ the product topology. Let

$$
\begin{aligned}
& \mathfrak{u}^{n}=\left\{V: V \in \mathfrak{u}, V_{l m}=0 \text { if } l+m>n\right\} \\
& u_{0}=\cup_{n} \mathfrak{u}^{n} .
\end{aligned}
$$

The above equation defines a topology in $\mathfrak{u}_{0}$ as an inductive limit of the spaces $\mathfrak{u}^{n}$.

Let $L(X, Y)$ be the set of continuous linear transformations from a vector space $X$ to a vector space $Y$.

Theorem 2.1. We have the following inclusions:

$$
\begin{aligned}
& \mathfrak{u}_{0} \subset L\left(\mathfrak{D}_{0}, \mathfrak{D}_{0}\right) \\
& \mathfrak{u}_{0} \subset L(\mathfrak{D}, \mathfrak{D}) \\
& \mathfrak{u} \subset L\left(\mathfrak{D}_{0}, \mathfrak{D}\right) .
\end{aligned}
$$

In each case the topology in $\mathfrak{u}_{0}$ or in $\mathfrak{u}$ is stronger than the corresponding topology of uniform convergence on bounded sets.

Proof. A bounded set $B$ is a set with the property that for any neighborhood $U$ of zero there corresponds a $\lambda>0$ with

$$
\lambda B \subset U .
$$

The bounded sets in $\mathfrak{D}_{0}$ are the sets which are bounded subsets of $\mathfrak{D}^{n}$, for some $n$. In view of this and the definition of the topology in $\mathfrak{D}_{0}$, we can replace the first and last inclusions to be proved by

$$
\begin{aligned}
& \mathfrak{u}_{0} \subset L\left(\mathfrak{D}^{n}, \mathfrak{D}_{0}\right) \\
& \mathfrak{u} \subset L\left(\mathfrak{D}^{n}, \mathfrak{D}\right),
\end{aligned}
$$

$n=1,2, \ldots$ Given a $\Phi$ in $\mathfrak{S}^{n}$, an integer $j \geqq 0$ and a $V$ in $\mathfrak{u}_{0}$ or in $\mathfrak{u}$,

$$
(V \Phi)_{j}
$$


depends continuously on $\varphi_{0}, \ldots, \varphi_{n}$ and if $V \in \mathfrak{u}^{k}$ for some given $k$, then

$$
V \Phi \in \mathfrak{S}^{n+k} \text {. }
$$

Thus the set theoretic inclusions (2.4) and (2.5) hold. The term (2.6) depends only on the $V_{l m}$ with $j-n \leqq l \leqq j, m \leqq n$. Let a bounded set $B$ in $\mathfrak{S}^{n}$ be given. (2.6) can be made small uniformly for $\Phi$ in $B$ by requiring that these $V_{l m}$ be sufficiently small. This proves (2.5). Let $U_{j}$ be a neighborhood of zero in the Schwartz space for the variables $k_{1}, \ldots, k_{j}$. The sets of the form

$$
U=\left\{\Phi: \varphi_{j} \in U_{j}, j=0,1, \ldots\right\}
$$

form a fundamental system of neighborhoods for $\mathfrak{S}_{\mathbf{0}}$. Given such a sequence $U_{j}$ we can find a sequence $U_{l m}$ such that

and

$$
V_{l m} \in U_{l m}
$$

$$
\Phi \in B
$$

imply

This proves (2.4).

$$
(V \Phi)_{j} \in U_{j}, j=1,2, \ldots
$$

Let $V$ be given in $\mathfrak{u}^{k}$ for some $k$. Then (2.6) depends on $\varphi_{n}$ only when $n \leqq j+k$. This proves the set theoretic inclusion (2.2). As a bounded set $B$ in $\mathfrak{P}$ we can take a set of the form

$$
B=\left\{\Phi: \varphi_{j} \in B_{j}\right\}
$$

where each $B_{j}$ is a bounded set in $\mathscr{S}$. Given a $j$ and a neighborhood $U_{j}$ of zero, we find a neighborhood $U_{l m}$ of zero such that

imply

$$
\varphi_{k} \in B_{k}, V_{l m} \in U_{l m}
$$

$$
(V \Phi)_{j} \in U_{j}
$$

This is possible since $(V \Phi)_{j}$ depends on a product

$$
V_{l m} \varphi_{k}
$$

only for $l \leqq j$ and $k-j \leqq m \leqq k$. This proves (2.2).

\section{$\S 3$. The structure of $\mathfrak{u}_{0}$ and $\mathfrak{u}$}

Theorem 3.1. Let $P, Q \in \mathfrak{u}_{0}$ and let $R \in \mathfrak{u}$. Then

$$
\begin{gathered}
P Q \in \mathfrak{u}_{0} \\
P R, R P \in \mathfrak{u} .
\end{gathered}
$$

It follows from this theorem that $\mathfrak{u}_{0}$ is an algebra and also a Lie algebra with the bracket

$$
[P, Q]=P Q-Q P
$$


Furthermore, for $R \in \mathfrak{u}$,

is defined.

$$
\text { ad } P(R)=[P, R]=P R-R P
$$

Definition. $\mathfrak{m}$ is a closed ideal in $\mathfrak{u}$ if ad $A \mathfrak{m} \subset \mathfrak{m}$ for each $A$ in $\mathfrak{u}_{0}$ and if $\mathfrak{m}$ is a closed subset of $\mathfrak{u}$.

Let $\mathfrak{z}=\left\{V: V \in \mathfrak{u}_{0}, V=V_{00}\right\}$. One can see that $\mathfrak{z}$ is a closed ideal in $\mathfrak{u}$, that $z$ is the center of $\mathfrak{u}_{0}$ and that the elements of $z$ act as multiples of the identity operator $I$ on the domains $\mathfrak{S}_{0}$ and $\mathfrak{P}$.

Theorem 3.2. $z$ is the only nontrivial closed ideal in $\mathfrak{u}$.

Proof of Theorem 3.1. A product $P_{j k} Q_{l m}$ does not have the right form to be in $\mathfrak{u}_{0}$ since $k$ annihilators from $P_{j k}$ precede $l$ creators from $Q_{l m}$. However, by use of the commutator identity

$$
\left[A-(k), A^{+}\left(k^{\prime}\right)\right]=\delta\left(k-k^{\prime}\right),
$$

we can interchange the order of an $A^{-}$and an $A^{+}$. Each such interchange leads to a new term with the $A^{+}$and $A^{-}$replaced by a $\delta$ function. If we perform the integration corresponding to the variables of the $\delta$ function the result is an operator with a smooth kernel. Thus $P Q \in \mathfrak{u}_{0}$, and more precisely we have proved

Lemma 3.3. For some choice of $S$ and $T$ in $\mathfrak{u}_{0}$ we have

where

$$
\begin{aligned}
P_{l_{1} m_{1}} Q_{l_{2} m_{2}} & =\sum_{0 \leqq r \leqq \min \left\{m_{1}, l_{2}\right\}} S_{l_{1}+l_{2}-r, m_{1}+m_{2}-r} \\
{\left[P_{l_{1} m_{1}}, Q_{l_{2} m_{2}}\right] } & =\sum_{1 \leqq r \leqq J} T_{l_{1}+l_{2}-r, m_{1}+m_{2}-r}
\end{aligned}
$$

$$
J=\max \left\{\min \left\{m_{1}, l_{2}\right\}, \min \left\{m_{2}, l_{1}\right\}\right\}
$$

Let $P \in \mathfrak{u}^{n}$ for some $n$ and let a $j$ and a $k$ be given. It follows from Lemma 3.3 that $\left(P R_{l m}\right)_{j k}=0=\left(R_{l m} P\right)_{j k}$ if $l+m>j+k+n$. Thus $(P R)_{j k}$ and $(R P)_{j k}$ are finite sums of elements of $\mathfrak{u}_{0}$ and so are in $\mathfrak{u}_{0}$. Hence $P R$ and $R P$ are in $\mathfrak{u}$.

Proof of Theorem 3.2. First we show that the closed ideal generated by any

$$
V_{01}=\int v(k) A^{-}(k) d k \quad(v \neq 0)
$$

is all of $\mathfrak{k}$. If $\varphi \in \mathscr{S}$ then one can compute

$$
\left[\int A^{+}(k) \bar{v}(k) \varphi\left(k^{\prime}\right) A^{-}(k) d k d k^{\prime}, v A-d k\right]=-\|v\|^{2} \int \varphi A-d k .
$$

Thus $\int \varphi A-d k$ is in the ideal generated by $\int v A-d k$. Now let $g_{l m}$ be a kernel with finite rank and let

$$
g_{l+1, m}=S y m_{k} \varphi\left(k_{1}\right) g\left(k_{2}, \ldots, k_{l+1}, k^{\prime}\right) .
$$

If $\varphi$ is suitably chosen then

$$
\left[G_{l+1, m}, \int \varphi A-d k\right]=-\|\varphi\|^{2} G_{l m},
$$

where the $G$ 's are the operators with kernels $g$. Thus the ideal contains 
all operators in $\mathfrak{u}_{0}$ whose kernels have finite rank and since this is dense in $\mathfrak{u}$, the ideal is all of $\mathfrak{u}$.

Similarly one proves that the closed ideal generated by $\int v A^{+} d k$ is $\mathfrak{u}$.

Now let $\mathfrak{m}$ be a closed ideal in $\mathfrak{u}$ which is not contained in $\mathfrak{z}$. We will prove that $\mathrm{m}$ contains an element of the form $\int v A^{ \pm} d k$ with $v \neq 0$. This will prove the theorem. Let $M$ be an element of $m$ which is not in $z$. Let

$$
N=\int A^{+}(k) \delta\left(k-k^{\prime}\right) A^{-}\left(k^{\prime}\right) d k d k^{\prime} .
$$

By a limiting procedure one proves that $[N, m] \subset m$. Furthermore for any polynomial $p$,

One computes that

$$
p(\operatorname{ad} N) \mathfrak{m} \subset \mathfrak{m}
$$

$$
\left[N, M_{l m}\right]=(l-m) M_{l m} .
$$

If we choose $p=p_{n, r}$ so that

then

$$
\begin{aligned}
& p(r)=1 \\
& p(k)=0 \text { if } k \in Z, k \neq r,|k| \leqq n,
\end{aligned}
$$

$$
\lim _{n} p_{n r}(\operatorname{ad} N) M=\sum_{l-m=r} M_{l m} \in \mathrm{m} .
$$

Thus we can suppose that $M$ has the form $\sum_{l-m=r} M_{l m}$ for some $r$. Each operator ad $\int v A^{ \pm} d k$ applied to $M$ removes an $A^{\mp}(k)$ from each term of $M$ and replaces it with a $\mp v(k)$. If products of the operators ad $\int v A^{ \pm} d k$ are applied to $M$, we can successively remove annihilationcreation operators from $M$ and for suitably chosen $v$ 's we achieve the following result. Either there is an $M=M_{10} \neq 0$ in $\mathrm{m}$ (and so the proof is finished) or there is an $M$ in $m$ of the form

$$
M=\sum_{l} M_{l, l+1} ; M_{01} \neq 0 \text {. }
$$

Let $\varphi$ be an element of $\mathscr{S}$ with unit norm and let $v_{l m}$ be a kernel. We can write $v_{l m}$ uniquely as a sum

$$
v_{l m}=\sum_{\alpha, \beta=0}^{l, m} \operatorname{Sym}_{k} \operatorname{Sym}_{k^{\prime}} \varphi^{-\alpha \otimes} v_{l-\alpha, m-\beta} \varphi^{\beta \otimes},
$$

where $v_{l-\alpha, m-\beta}$ is a kernel orthogonal to $\varphi$ in the following sense:

$$
\begin{aligned}
0 & =\int \varphi\left(k_{1}\right) v_{l-\alpha, m-\beta}\left(k_{1}, \ldots, k^{\prime}\right) d k_{1} \\
& =\int \varphi\left(k_{1}^{\prime}\right) v_{l-\alpha, m-\beta}\left(k_{1}, \ldots, k_{m-\beta}^{\prime}\right) d k_{1}^{\prime},
\end{aligned}
$$

and where $\varphi^{\alpha \otimes}=\varphi \otimes \cdots \otimes \varphi$ is an $\alpha$-fold tensor product. Let $v_{l m}^{\alpha \beta}$ be the $\alpha, \beta$ term in this sum and let $V_{l m}^{\alpha \beta}$ be the corresponding operator. Let

One can compute

$$
P(\varphi)=\int A^{+} \bar{\varphi} \otimes \varphi A^{-} d k d k^{\prime} .
$$

$$
\left[P(\varphi), V_{l m}^{\alpha \beta}\right]=(\alpha-\beta) V_{l m}^{\alpha \beta} .
$$


We choose $\varphi$ proportional to $m_{0}$, the kernel of $M_{01}$. We use the $p_{n,-1}$ defined above. We have

$$
\lim _{n} p_{n,-1}(\operatorname{ad} P(\varphi)) M=\sum M_{l, l+1}^{\alpha, \alpha+1} \in \mathrm{m} .
$$

Since $M_{01}=M_{01}^{01}$, we can suppose that $M$ has the form

$$
M=\sum M_{l, l+1}^{\alpha, \alpha+1} ; \quad M_{01} \neq 0 .
$$

We return to a general $\varphi$ and we let

$$
\begin{aligned}
& B=\operatorname{ad} \int \varphi \otimes \varphi A^{-} A^{-} d k^{\prime} \operatorname{ad} \int A^{+} A^{+} \bar{\varphi} \otimes \bar{\varphi} d k \\
& C=\operatorname{ad} \int \varphi A^{-} d k^{\prime} \operatorname{ad} \int A^{+} \bar{\varphi} d k .
\end{aligned}
$$

One can compute

$$
\begin{aligned}
{\left[B, V^{\alpha \beta}\right]=4\left\{-(\alpha+1) \beta V^{\alpha \beta}+\alpha \beta(\beta-\alpha-2) W^{\alpha-1, \beta-1}+\right.} & \\
& \left.+\alpha(\alpha-1) \beta(\beta-1) X^{\alpha-2, \beta-2}\right\} .
\end{aligned}
$$

Here $V=V_{l m}$ has its kernel given by (3.2) and $W^{\alpha-1, \beta-1}$ and $X^{\alpha-2, \beta-2}$ have kernels

Also

$$
\begin{aligned}
& w^{\alpha-1, \beta-1}=\operatorname{Sym}_{k, k^{\prime}} \varphi^{(\alpha-1) \otimes} v_{l-\alpha, m-\beta} \varphi^{(\beta-1) \otimes} \\
& x^{\alpha-2, \beta-2}=\operatorname{Sym}_{k, k^{\prime}} \varphi^{(\alpha-2) \otimes} v_{l-\alpha, m-\beta} \varphi^{(\beta-2) \otimes} .
\end{aligned}
$$

$$
\begin{aligned}
{\left[C, V^{\alpha \beta}\right] } & =\alpha \beta W^{\alpha-1, \beta-1} \\
{\left[C,\left[C, V^{\alpha \beta}\right]\right] } & =\alpha(\alpha-1) \beta(\beta-1) X^{\alpha-2, \beta-2}
\end{aligned}
$$

It follows that for some linear combination $D$ of ad $B$, ad $C$ and $(\operatorname{ad} C)^{2}$, we have

$$
\begin{aligned}
D M & =\sum(\alpha+1)^{2} M_{l, l+1}^{\alpha, \alpha+1} \in \mathrm{m} . \\
\lim _{n} p_{n, 1}(D) M & =\sum M_{l, l+1}^{01} \in \mathrm{m} .
\end{aligned}
$$

We write $m_{l, l+1}^{01}=\operatorname{Sym}_{k^{\prime}} q_{l, l} \otimes m_{01}$, for some kernels $q_{l, l}$ which are orthogonal to $m_{01}$. We choose an orthonormal base $\varphi_{0}, \varphi_{1}, \ldots$ of $\mathscr{S}$ consisting of Hermite functions. Without loss of generality $m_{01}=\varphi_{0}$. The kernels $q_{\imath \iota}$ can be written as an infinite series of tensor products of the $\varphi_{j}$ with convergence in $\mathscr{S}$, see $[5, \S 2]$ for example. Thus if we choose an integer $K=K(L)$ sufficiently large, we will have

$$
q_{l l}=r_{l l}+s_{l l}, \text { all } l \leqq L
$$

where $s_{l l}$ is small in $\mathscr{S}$, each term of $r_{l l}$ contains at least one factor from the set

$$
\left\{\varphi_{1}, \ldots, \varphi_{K}\right\},
$$

and where $s_{l l}$ is independent of $\varphi_{1}, \ldots, \varphi_{K}$ (and of $\varphi_{0}$ also). 
The expansion of the kernels leads to a corresponding expansion of the $M_{l, l+1}^{01}$. Each such term is an eigenvector for ad $P\left(\varphi_{j}\right)$ with eigenvalue

\{number of times $\varphi_{j}$ occurs in the first $l$ variables\}-

- number of times $\varphi_{i}$ occurs in the last $l+1$ variables .

Since ad $P\left(\varphi_{j}\right) M_{01}=0$, we can argue as above and eliminate those terms for which the eigenvalue is not zero. We do this for $1 \leqq j \leqq K$.

We set $\varphi=\varphi_{j}$ in our definition of $B=B_{j}$ and $C=C_{j}$. For a new linear combination $E_{j}$ of $\operatorname{ad} B_{j}, \operatorname{ad} C_{j}$ and $\left(\operatorname{ad} C_{j}\right)^{2}$ we have each term in the expansion of the $M_{l, l+1}^{01}$ an eigenvector of $E_{j}$ with eigenvalue

As above

$$
\begin{aligned}
& \geqq 1 \text { if } \varphi_{j} \text { occurs } \\
& =0 \text { if } \varphi_{j} \text { does not occur . }
\end{aligned}
$$

$$
\left[\prod_{j=1}^{K} \lim _{n} p_{n, 0}\left(E_{j}\right)\right] M_{l l+1}^{01}=s_{l l} \otimes \varphi_{0}
$$

for $1 \leqq l \leqq L$. Also $p_{n, 0}\left(E_{j}\right) M_{01}=M_{01}$. It follows that $M_{01} \in \mathrm{m}$ and the proof is complete.

Our Hamiltonians $H=H_{0}+V$ and $H_{0}$ are not in $\mathfrak{u}$ since the kernel of $H_{0}$ is singular. However the set

$$
\left\{\lambda H_{0}\right\}+\mathfrak{u}=\mathfrak{w}
$$

is a Lie algebra since the bracket $\left[H_{0}, V\right]$ is defined and is in $\mathfrak{u} ; \mathfrak{u}$ is thus an ideal in $\mathfrak{w}$. It can be seen that the algebra $\mathfrak{a}$ of operators of the form

$$
\lambda H_{0}+V_{00}
$$

is a maximal abelian subalgebra of $\mathfrak{w}$. We remark that Theorem 3.2 and its proof remain valid if we replace the requirement that the kernels belong to $\mathscr{S}$ by the requirement that they belong to $L_{2}$.

\section{$\S$ 4. Canonical transformations of $\mathfrak{u}$}

The constructions of automorphisms and derivations which we shall consider lead to operators in $\mathfrak{u}$ which are not in $\mathfrak{u}_{0}$, even if our perturbation $V$ is in $\mathfrak{u}_{0}$. Thus $\mathfrak{u}_{0}$ is too small to provide a satisfactory framework for the theory. On the other hand $\mathfrak{u}$ is not a Lie algebra and we will show in this section that an attempt to study derivations and automorphisms of $\mathfrak{u}$ leads to certain pathological phenomena.

We consider the following explicitly soluble problem. Let $V=\sum_{m>0} V_{0 m}$ consist entirely of annihilation operators. Let $\Gamma V=\sum \Gamma V_{0 m}$, where $\Gamma V_{0 m}$ has the kernel

$$
-\left(\Sigma \omega_{j}\right)^{-1} v_{0 m} .
$$

Then $\Gamma V \in \mathfrak{u}$ and $\Gamma V$ is a solution of the equation

$$
\left[H_{0}, \Gamma V\right]=V \text {; }
$$


also $V$ and $\Gamma V$ commute. Using this, it can be seen that

We let

$$
\begin{aligned}
& \exp (\Gamma V)\left(H_{0}+V\right) \exp (-\Gamma V) \\
& =\exp (\Gamma V) H_{0} \exp (-\Gamma V)+V \\
& =H_{0}+\left[\Gamma V, H_{0}\right]+V \\
& =H_{0} .
\end{aligned}
$$

$$
\begin{aligned}
\mathfrak{a}_{V} & =\{\lambda H\}+V_{00} \\
& =\exp (-\Gamma V) \mathfrak{a} \exp (\Gamma V) .
\end{aligned}
$$

Since $\mathfrak{a}$ is a maximal abelian subalgebra of $\mathfrak{w}$, one might expect that $\mathfrak{a}_{V}$ would be maximal abelian also. However we will see that this is not the case. We let

$$
\begin{aligned}
B^{+}(k) & =\exp (\Gamma V) A^{+}(k) \exp (-\Gamma V) \\
& =A^{+}(k)+\left[\Gamma V, A^{+}(k)\right] \\
B^{-}(k) & =\exp (\Gamma V) A^{-}(k) \exp (-\Gamma V)=A^{-}(k) .
\end{aligned}
$$

One might hope that the map

$$
\begin{aligned}
G & =G\left(A^{ \pm}\right) \rightarrow G\left(B^{ \pm}\right) \\
& =G\left(A^{+}+\left[\Gamma V, A^{+}\right], A^{-}\right)
\end{aligned}
$$

obtained by substituting $B^{ \pm}$for $A^{ \pm}$would be an automorphism of $\mathfrak{u}$. Although $\exp ( \pm \Gamma V)$ and $B^{ \pm}$are defined (since $\left.V=\sum_{m} V_{0 m}\right), G\left(B^{ \pm}\right)$ is not in general defined. By this we mean that formally

$$
G\left(B^{ \pm}\right)=F=\Sigma_{l m} F_{l m}
$$

and that each kernel $f_{l_{m}}$ is expressed as an infinite sum of terms depending linearly on the kernels $g_{l^{\prime} m^{\prime}}$. Since there is no restriction in the rate of growth of the $g_{l^{\prime} m^{\prime}}$ as $l^{\prime}, m^{\prime} \rightarrow \infty$, such a sum will not in general converge.

Definition. Let $g=g\left(k_{1}, \ldots, k_{p}\right)$ be a distribution of class $\mathscr{S}^{*}$ which is symmetric in the variables $k_{1}, \ldots, k_{p}$. If $v_{l+p, m}=v_{l+p, m}$ $\left(k_{1}, \ldots, k_{l+p}, k_{1}^{\prime}, \ldots, k_{m}^{\prime}\right)$ is a kernel of the class we have considered (§2), let

$$
\begin{aligned}
\left\langle g, v_{l+p, m}\right\rangle\left(k_{p+1},\right. & \left.\ldots, k_{l+p}, k^{\prime}\right) \\
& =\int \bar{g}\left(k_{1}, \ldots, k_{p}\right) v_{l+p, m}\left(k_{1}, \ldots, k_{l+p}, k^{\prime}\right) d k_{1} \ldots d k_{p} .
\end{aligned}
$$

Theorem 4.1. Let $g$ be given as above. If $g \neq 0$, then the range of the map

$$
v_{l+p, m} \rightarrow\left\langle g, v_{l+p, m}\right\rangle
$$

is the set of all our kernels in the variables $k_{1}, \ldots, k_{l}, k_{1}^{\prime}, \ldots, k_{m}^{\prime}$. 
Proof. It is clear that $\left\langle g, v_{l+p, m}\right\rangle$ is a kernel of the right class. Let $\varphi$ be a function of one variable $k_{1}, \varphi \in \mathscr{S}$, such that

$$
0 \neq \int \bar{g}\left(k_{1}, \ldots, k_{p}\right) \varphi\left(k_{1}\right) \ldots \varphi\left(k_{p}\right) d k_{1} \ldots d k_{p} .
$$

Let a kernel $v_{l m}$ be given. There are kernels $u_{0 m}, \ldots, u_{l m}$ for which

$$
v_{l m}=\sum_{j=0}^{l} \varphi^{(l-j) \otimes} \otimes u_{j m} .
$$

Here $\varphi^{k \otimes}$ is the $k$-fold tensor product of $\varphi$ (and $\left\langle\varphi, u_{j m}\right\rangle=0$ ). All tensor products are taken to be symmetric in the first $l$ variables. Any kernel of the form

$$
\varphi^{l \otimes} \otimes u_{0 m}=\text { const. }\left\langle g, \varphi^{(l+p) \otimes} \otimes u_{0 m}\right\rangle
$$

is in the range of (4.1). We suppose by induction on $J$ that for $j<J$ we have kernels of the form $\varphi^{(l-j) \otimes} \otimes u_{j m}$ in the range of (4.1). Then

$$
\begin{aligned}
\left\langle g, \varphi^{(l-J+p) \otimes} \otimes u_{J m}\right\rangle= & \left\langle g, \varphi^{p \otimes}\right\rangle \varphi^{(l-J) \otimes} \otimes u_{J m} \\
& +(\text { terms in the range of }(4.1))
\end{aligned}
$$

and so $\varphi^{(l-J) \otimes} \otimes u_{J m}$ is in the range of (4.1). This completes the induction and the proof.

Suppose that $\varphi$ has $L_{2}$ norm equal to one. The $u_{j m}$ are given explicitly by a formula

$$
\text { const. } u_{j m}=\left\langle\varphi^{(l-j) \otimes}, v_{l m}\right\rangle-\sum_{i=0}^{j-1} \text { const. } \varphi^{(j-i) \otimes} \otimes u_{i m}
$$

where the constants are ratios of the factorials. It follows that the $u_{j m}$ depend in a linear continuous fashion on $v_{l m}$. Thus $v_{l+p, m}$ can be chosen to be a continuous linear function of $v_{l m}$.

If $g$ and $v_{l m}$ are in the class $L^{\wedge}$ of rapidly decreasing continuous functions introduced in $\S 8$, then $\varphi$ can be chosen in this class also. The $u_{j m}$ and $v_{l+p}$ belong to this class (this requires the use of formula (8.10)), and $v_{l+p}$ can again be chosen to be a linear continuous function of $v_{l m}$. We need these facts in $\S 9$.

Theorem 4.2. Let

$$
H=H_{0}+\sum_{l-m>-r} V_{l m}+V_{0 r}
$$

for some $r$ with $r \geqq 2$. Suppose that $V_{0 r} \neq 0$. There is a $G$ in $\mathfrak{u}$ for which

$$
\begin{aligned}
{[H, G] } & =0, \\
G & \neq G_{00} .
\end{aligned}
$$

Proof. We write $G=\sum_{k>0} G_{k}$, where $G_{l}=\sum_{l-m=k} G_{l m}$. The kernel of $\left[H_{0}, G_{l m}\right]$ is $\left(\sum \omega\right) g_{l m}$, where

$$
\sum \omega=\sum_{i=1}^{l} \omega\left(k_{i}\right)-\sum_{j=1}^{m} \omega\left(k_{j}^{\prime}\right)
$$

The equation for $G$ can be written as

$$
\left[V_{0 r}, G_{k}\right]=\text { terms depending on } G_{1}, \ldots, G_{k-1}
$$

for $k=1,2, \ldots$ We solve this by induction on $k$. For fixed $k,(4.3)$ is 
equivalent to

or to

$$
\left[V_{0 r}, G_{k}\right]_{j+k-r, j}=\text { known operator }
$$

$$
\begin{aligned}
{\left[V_{0 r}, G_{j+k, j}\right]_{j+k-r, j}=} & \text { known operator } \\
& + \text { terms depending on } G_{k, 0}, \ldots, G_{j-1+k, j-1},
\end{aligned}
$$

for $j \geqq 0, j \geqq r-k$. We solve this by induction on $j$. Then (4.4) is equivalent to

$$
\left\langle\bar{v}_{0 r}, g_{j+k, j}\right\rangle=\text { known kernel } .
$$

This can be solved by Theorem 4.1. Finally we observe that $G_{10}$, for example, can be chosen arbitrarily. Thus we can have $G \neq G_{00}$.

\section{§ 5. A Lie algebra between $\mathfrak{u}_{0}$ and $\mathfrak{u}$}

Let $\mathfrak{u}_{1 r}$ be the set of $Q$ in $\mathfrak{u}$ which have the form

$$
Q=\sum_{l-m \geqq r} Q_{l m}
$$

$\mathfrak{u}_{1 r}$ is a closed subset of $\mathfrak{u}$; we give it the relative topology from $\mathfrak{u}$. Let

$$
\mathfrak{u}_{1}=\cup_{r} \mathfrak{u}_{1 r}
$$

and let $\mathfrak{u}_{1}$ have the inductive limit topology.

Theorem 5.1. We have the following inclusion:

$$
\mathfrak{u}_{1} \subset L(\mathfrak{O}, \mathfrak{D}) \text {. }
$$

The topology of $\mathfrak{u}_{1}$ is stronger than the topology of uniform convergence on bounded sets.

Theorem 5.2. $\mathfrak{u}_{1}$ is a subalgebra of $L(\mathfrak{D}, \mathfrak{D})$ and the product $P Q$ of two elements of $\mathfrak{u}_{1}$ is jointly continuous in its two factors $P$ and $Q$ provided one of the factors $P$ or $Q$ is required to remain in a bounded set.

The algebra $\mathfrak{u}_{1}$ gives an unsymmetrical preference to creation operators. If we formed an analogous algebra but gave the preference to annihilation operators instead, we could obtain the set of formal adjoints $\mathfrak{u}_{1}^{*}$ to $\mathfrak{u}_{1}$. Here we have

$$
\mathfrak{u}_{1}^{*} \subset L\left(\mathfrak{S}_{0}, \mathfrak{D}_{0}\right) .
$$

Proof of Theorem 5.1. Let $B$ be a bounded set in $D$. We can suppose that $B$ has the form

$$
B=\left\{\Phi: \varphi_{j} \in B_{j}\right\}
$$

where each $B_{j}$ is a bounded set in an $\mathscr{S}$ space. For any given $k$ and for $Q$ in $\mathfrak{u}_{1 r}$, the component $(Q \Phi)_{k}$ depends only on

$$
\varphi_{0}, \ldots, \varphi_{k+|r|}
$$

and on $Q_{l m}$ for $l \leqq k, m \leqq k+|r|$. Thus if these terms of $Q$ are sufficiently small in $\mathscr{S}$ and if $\Phi \in B$, then $(Q \Phi)_{k}$ can be made as small as desired. In view of the definition of the topology in $\mathfrak{u}_{1}$, this proves the theorem. 
Proof of Theorem 5.2. It is sufficient to prove separate continuity and also joint continuity at zero. To prove separate continuity, it is sufficient to consider the case $P \in \mathfrak{u}_{1 r}$, for some fixed $r$. Then a term $(P Q)_{l m}$ depends only on

and on

$$
\left\{P_{j k}: j \leqq l, k \leqq l+|r|\right\}
$$

$$
\left\{Q_{j k}: j \leqq 2 l+|r|, k \leqq m\right\}
$$

by Lemma 3.3. Thus $P Q \in \mathfrak{u}$ and the product is separately continuous. By Lemma 3.3,

$$
\mathfrak{u}_{1 r} \mathfrak{u}_{1 s} \subset \mathfrak{u}_{1 r+s}
$$

and so $\mathfrak{u}_{1}$ is a subalgebra of $L(\mathfrak{D}, \mathfrak{D})$. The bounded sets in $\mathfrak{u}_{1}$ are just the sets which are bounded subsets of $\mathfrak{u}_{1 r}$ for some $r$. Thus if $P$ is in some bounded set, the above argument shows the joint continuity at zero.

Now let $B$ be a bounded set in some $\mathfrak{u}_{1 r}$ and suppose that $Q \in B$. We can suppose that $B$ has the form

$$
\left\{Q: Q \in \mathfrak{l}_{1 r}, Q_{l m} \in B_{l_{m}}\right\}
$$

where the $B_{l m}$ are bounded subsets of $\mathscr{S}$ spaces. To give a neighborhood $U$ of zero in $\mathfrak{u}_{1}$ we choose an integer $k_{0}$. For each $k \geqq k_{0}$ we choose an integer $l(k) \geqq l(k-1)$ and we choose neighborhoods $U_{l m}$. We define $U$ to be the set

$$
\left\{R: R \in \mathfrak{u}_{1}, R_{l, l+k} \in U_{l, l+k} \text { for } k \geqq k_{0} \text { and } l \leqq l(k)\right\} .
$$

These $U$ form a basis for neighborhoods of zero in $\mathfrak{u}_{1}$. A term $P_{\alpha, \alpha+\beta}$ contributes to a term $(P Q)_{l, l+k}$ only when $k \leqq \beta+r$ and $\alpha \leqq l$. The formulas

$$
\left(P_{\alpha, \alpha+\beta} Q\right)_{l, l+k} \in 2^{-\alpha-|\beta|} U_{l, l+k}, \quad l \leqq l(k)
$$

will be satisfied for $k \geqq k_{0}$ provided each of the terms

$$
\left\{P_{\alpha, \alpha+\beta}: \beta \geqq k_{0}-r, \alpha \leqq l(\beta+r)\right\}
$$

is sufficiently small. These terms will be small if $P$ is a suitable neighborhood $V$ of zero and this completes the proof of the theorem.

Let $\mathfrak{w}_{1}$ be the set of operators of the form $H=\lambda H_{0}+V, V \in \mathfrak{u}_{1}$. $\mathfrak{w}_{1}$ is also a Lie algebra. Theorem 3.2 and its proof are valid for $\mathfrak{u}_{1}$; consequently $\mathfrak{u}_{1} / \mathfrak{z}$ is simple. Each subspace $\mathfrak{u}_{1 r}, 0 \leqq r$, of $\mathfrak{u}_{1}$ is a subalgebra and a Lie subalgebra of $\mathfrak{u}_{1}$. This is not the case for $r<0$. If $0 \leqq r_{1} \leqq r_{2}$, then $\mathfrak{u}_{1 r_{2}}$ is an ideal in $\mathfrak{u}_{1 r_{1}}$. The derived subalgebra of $\mathfrak{u}_{1 r}$ is contained in $\mathfrak{u}_{12 r}$ for $0 \leqq r$.

Theorem 5.3. If $Q \in \mathfrak{u}_{1 r}$ and if $0 \leqq r$, then the power series $\exp Q$ converges in the topology of $\mathfrak{u}_{1}$ to an element of $I+\mathfrak{u}_{1}$. The set

$$
\left\{\sum_{n \leqq N}(n !)^{-1} Q^{n}: N=0,1, \ldots\right\}
$$

of partial sums is a bounded set. 
Theorem 5.4. If $Q \in \mathfrak{u}_{1 r}$, if $1 \leqq r$ and if $\left\{a_{0}, a_{1}, \ldots\right\}$ is any sequence of numbers, then the power series

converges.

$$
\sum a_{i} Q^{i}
$$

Proof of Theorem 5.4. Let $l$ and $m$ be given and let $Q$ and $r$ be as in the theorem. Then $\left(Q^{n}\right)_{l m}=0$ if $n>l-m$.

Proof of Theorem 5.3. A product

$$
Q_{l_{1} m_{1}} Q_{l_{2} m_{2}}
$$

has a nonzero $l m$ term only if

Hence

$$
\begin{aligned}
& l_{1} \leqq l \\
& l_{2} \leqq l_{2}+\left(l_{1}-m_{1}\right) \leqq l .
\end{aligned}
$$

$$
\left(Q^{n}\right)_{l m}=\left(\left(\sum_{m_{1} \leqq l_{1} \leqq l} Q_{l_{1} m_{1}}\right)^{n}\right)^{l m}
$$

and so there are at most $(2 l)^{n}$ terms of the form

$$
\left(Q_{l_{1} m_{1}} \ldots Q_{l_{n} m_{n}}\right)_{l m}
$$

which contribute to $\left(Q^{n}\right)_{l m}$. Each of these terms is a sum

$$
\left.\sum_{0 \leqq j_{1}, \ldots, j_{n} \leqq l}\left(\ldots\left(Q_{l_{1} m_{1}} \underset{j_{1}}{\stackrel{\circ}{j_{1}}}-Q_{l_{2} m_{2}}\right) \underset{j_{2}}{-\circ} \ldots\right) \underset{j_{n}}{\circ}-Q_{l_{n} m_{n}}\right)_{l m} .
$$

Here -0 - is defined by the formula

$$
Q_{l_{1} m_{1}}-\underset{j}{\circ}-Q_{l_{2} m_{2}}=\left(Q_{l_{1} m_{1}} Q_{l_{2} m_{2}}\right)_{l_{1}+l_{3}-j, m_{1}+m_{2}-j},
$$

which differs slightly from the definition in [2]. There are $l^{n}$ summands in (5.1) and so there are $\left(2 l^{2}\right)^{n}$ terms in $\left(Q^{n}\right)_{l m}$ which have the form of a summand in (5.1). Hence for any seminorm $\|\cdot\|$ defined on the operators $Q_{l m}$ we can find a constant $K$ such that

$$
\left\|\left(Q^{n}\right)_{l m}\right\| \leqq K^{n} .
$$

The convergence of $\exp Q$ and the boundedness of the partial sums follow from this.

Let $H \in \mathfrak{w}_{1}$ and let $Q \in \mathfrak{u}_{1,0}$. The map

$$
H \rightarrow \exp (Q) H \exp (-Q)
$$

is an automorphism of $\mathfrak{w}_{1}$. This map is determined by its effect on the $A^{ \pm}$. Let

Then

$$
B^{ \pm}=\exp (Q) A^{ \pm} \exp (-Q) .
$$

$$
\begin{gathered}
\exp (Q) V \exp (-Q)=\sum_{l m} \int B^{+}\left(k_{1}\right) \ldots B^{+}\left(k_{l}\right) v_{l m}\left(k, k^{\prime}\right) B^{-}\left(k_{1}^{\prime}\right) \\
\ldots B^{-}\left(k_{m}^{\prime}\right) d k d k^{\prime}
\end{gathered}
$$


The conventional relation between automorphisms and derivations holds for (5.2).

Theorem 5.5. Let $H$ be in $\mathfrak{w}_{1}$ and let $Q$ be in $\mathfrak{u}_{1,0}$. Then

$$
\exp (Q) H \exp (-Q)=\sum_{n=1}^{\infty}(n !)^{-1}(\operatorname{ad} Q)^{n} H .
$$

The series converges in $\mathfrak{w}_{1}$.

Proof. We have

$\exp (Q) H \exp (-Q)=\lim _{J, K \rightarrow \infty} \sum_{j \leqq J, k \leqq K}(j ! k !)^{-1} Q^{j} H(-Q)^{k}$.

Because the partial sums $\sum_{j \leqq J}(j !)^{-1}( \pm Q)^{k}$ are bounded, we can write this limit as

$\lim _{N \rightarrow \infty} \sum_{n \leqq N}(n !)^{-1} \sum_{j+k=n}\left(\begin{array}{c}n \\ j\end{array}\right) Q^{j} H(-Q)^{k}$

This proves the theorem.

$$
=\lim _{N \rightarrow \infty} \sum_{n \leqq N}(n !)^{-1}(\operatorname{ad} Q)^{n} H .
$$

In a similar fashion one proves

$$
\exp (s Q) \exp (t Q)=\exp ((s+t) Q) .
$$

The group $I+\mathfrak{u}_{1,1}$ is the (infinite dimensional Lie) group corresponding the to Lie algebra $\mathfrak{u}_{1,1}$. This group and its Lie algebra are in some sense nilpotent, although they are not nilpotent if one uses the conventional definitions [3]. The next theorem gives a property which they have in common with nilpotent lie groups and algebras.

Theorem 5.6. The mapping exp is a one-one map of $\mathfrak{u}_{1,1}$ onto $I+\mathfrak{u}_{1,1}$. Proof. Let $V$ be in $\mathfrak{u}_{1,1}$ and let

$$
V_{r}=\sum_{l-m=r} V_{l m} \text {. }
$$

We must find a solution $Q \in \mathfrak{u}_{1,1}$ of the equation

$$
\exp Q=I+V \text {. }
$$

This is equivalent to solving the sequence of equations

$$
Q_{r}=V_{r}-\sum_{n \geqq 2} n !^{-1}\left(Q^{n}\right)_{r}
$$

for $r=1,2, \ldots$. For a $Q$ in $\mathfrak{u}_{1,1}$ the operator

$\left(Q^{n}\right)_{r}$

depends only on $Q_{1}, \ldots, Q_{r-n+1}$. Thus the equations (5.6) can be solved by induction, and the solution is unique. This proves the theorem.

Let an $r$ be given. There is a polynomial $p_{r}$ such that

$$
\sum_{j \leqq r} Q_{j}=\sum_{j \leqq r} p_{r}(V)_{j} .
$$

This can be proved directly by induction on $r$. It also follows from the Baker-Hausdorff formula [3]. This formula exhibits $p_{r}$ explicitly and in fact gives $Q$ as a power series in $V$. 
Let $H$ be an element of $\mathfrak{w}_{1}$ and let

$$
\mathfrak{c}_{H}=\left\{Q: Q \in \mathfrak{u}_{1,1},[Q, H]=0\right\} .
$$

Theorem 5.7. $\mathfrak{c}_{H}$ is a subalgebra of $\mathfrak{u}_{1,1}$ and $I+\mathfrak{c}_{H}$ is a subgroup of $I+\mathfrak{u}_{1,1}$. Also

$$
\begin{aligned}
I+\mathfrak{c}_{H} & =\exp \mathfrak{c}_{H} \\
& =\left\{Q: Q \in I+\mathfrak{u}_{1,1},[Q, H]=0\right\} .
\end{aligned}
$$

Proof. If $Q \in \mathfrak{c}_{H}$ then $\left[Q^{n}, H\right]=0$ and $Q^{n} \in \mathfrak{c}_{H}$. Thus $\mathfrak{c}_{H}$ is an algebra, $I+\mathfrak{c}_{H}$ is a group and

$$
\exp \mathfrak{c}_{H} \subset I+\mathfrak{c}_{H} .
$$

The reverse inclusion follows from the fact that the $Q$ in (5.5) is a limit of polynomials (without constant terms) in $V$. The last equality is obvious.

We remark that $\mathfrak{c}_{H}$ and $I+\mathfrak{c}_{H}$ are closed in $\mathfrak{u}$.

\section{§ 6. Canonical forms for certain elements of $\mathfrak{w}_{1}$}

In this section we consider elements $H$ of $\mathfrak{w}_{1}$ of the form

$$
\begin{gathered}
H=\lambda H_{0}+\sum_{l-m>-r} V_{l m}+V_{0 r} \\
r>0, \quad V_{0 r} \neq 0 .
\end{gathered}
$$

The main result is

Theorem 6.1. If $H$ is given by (6.1), (6.2) then there is a $Q$ in $\mathfrak{u}_{1,1}$ for which

$$
\exp (Q) H \exp (-Q)=\lambda H_{0}+V_{0 r} .
$$

It can be seen that the equations

$$
\begin{gathered}
\exp (Q)\left(\lambda H_{0}+S_{0 r}\right) \exp (-Q) \\
=\mu H_{0}+T_{0 r}, \\
Q \in u_{1,1}
\end{gathered}
$$

imply $\mu=\lambda$ and $S_{0 r}=T_{0 r}$. Thus we regard

$$
H_{1}=\lambda H_{0}+V_{0 r}
$$

as a canonical form for $H$ with respect to the inner automorphism grour $\exp \left(\mathfrak{u}_{1,1}\right)$. In proving this theorem we do not assume $\lambda \neq 0$, and we regard $V_{0 r}$ rather than $H_{0}$ as the dominant term in $H$.

Proof of Theorem 6.1. We write

where

$$
Q=\sum_{s=0}^{\infty} Q_{s}
$$

and

$$
Q_{s}=\sum_{l-m=s} Q_{l m}, \quad s>0
$$

$$
Q_{0}=0
$$


and we let

$$
Q^{(t)}=\sum_{s \leqq t} Q_{s}
$$

Let a $t \geqq 0$ be given. We suppose inductively that $Q_{0}, Q_{1}, \ldots, Q_{t}$ have been chosen and that

$$
\sum_{n=0}^{\infty}(n !)^{-1}\left(\operatorname{ad} Q^{(t)}\right)^{n} H \equiv H_{1} \quad\left(\bmod \mathfrak{u}_{1,-r+t+1}\right) .
$$

The inductive hypothesis is true for $t=0$; we use the hypothesis and define $Q_{t+1}$. Let

$\sum_{n=0}^{\infty}(n !)^{-1}\left(\operatorname{ad} Q^{(t)}\right)^{n} H \equiv H_{1}+\sum_{l-m=-r+t+1} V_{l m}(t)\left(\bmod \mathfrak{u}_{1,-r+t+2}\right)$.

We choose $Q_{t+1}$ as a solution of the equation

$$
\left[Q_{t+1}, V_{0 r}\right]=-\sum_{l-m=-r+t+1} V_{l m}(t) .
$$

This equation is equivalent to the system of equations

$$
\sum_{j=1}^{r} V_{0 r} \underset{j}{\circ-} Q_{m+j-r+t+1, m+j}=V_{m-r+t+1, m}(t) .
$$

We solve (6.5) by induction on $m$. The equation for $Q_{m+t+1, m}$ then has the form

$$
V_{0 r} \longrightarrow Q_{m+t+1, m}=\text { known function . }
$$

This equation has solutions, by Theorem 4.1. The induction starts with $m=\max \{0, r-t-1\}$, and for $0 \leqq m<r-t-1, Q_{m+t+1, m}$ can be chosen arbitrarily.

However

$$
\left(\operatorname{ad} Q^{(t)}\right)^{n} H \equiv\left(\operatorname{ad} Q^{(t+1)}\right)^{n} H \quad\left(\bmod \mathfrak{u}_{1,-r+t+2}\right)
$$

for $n>1$. Thus

$$
\sum_{n=0}^{\infty}\left(\operatorname{ad} Q^{(t+1)}\right)^{n} H \equiv H_{1} \quad\left(\bmod \mathfrak{u}_{1,-r+t+2}\right),
$$

and the induction on $t$ is complete. We define $Q$ by (6.4). As above

and

$$
\left(\operatorname{ad} Q^{(t)}\right)^{n} H \equiv(\operatorname{ad} Q)^{n} H \quad\left(\bmod \mathfrak{u}_{1,-r+t+1}\right),
$$

$$
\sum_{n=0}^{\infty}(n !)^{-1}(\operatorname{ad} Q)^{n} H \equiv H_{1} \quad\left(\bmod \mathfrak{u}_{1,-r+t+1}\right) .
$$

This is true for $t=1,2, \ldots$ and this completes the proof.

\section{\$ 7. The transformation and solution of the Schrödinger equation}

In this section we consider time dependent operators. We set

$$
A^{ \pm}(k, t)=e^{\mp i \omega t} A^{ \pm}(k, 0),
$$

where the $A^{ \pm}(k, 0)$ are the standard annihilation creation operators introduced in $\S 2$. We consider operators of the form

$$
\begin{gathered}
V_{l m}=\int A^{+}\left(k_{1}, t\right) \ldots A^{+}\left(k_{l}, t\right) v_{l m}\left(k, k^{\prime}\right) A^{-}\left(k_{1}^{\prime}, t\right) \ldots A^{-}\left(k_{m}^{\prime}, t\right) d k d k^{\prime}, \\
\text { Commun. math. Phys., Vol.2 }
\end{gathered}
$$


and of the form

$$
V=\Sigma V_{l m}
$$

Such operators are said not to depend explicitly on $t$. We see that the $A^{ \pm}$ satisfy the differential equation

$$
i \frac{\partial}{\partial t} A^{ \pm}(k, t)=\left[H_{0}, A^{ \pm}(k, t)\right] .
$$

Let

$$
\Phi(t)=\exp \left(-i t H_{0}\right) \Phi(0)
$$

be a solution of the free field Schrödinger equation

$$
i \frac{\partial}{\partial t} \Phi=H_{0} \Phi \text {. }
$$

If $\Phi(0) \in \mathfrak{S}_{0}$ then $\Phi(t) \in \mathfrak{S}_{0}$ for all $t$ and $(\partial / \partial t) \Phi$ exists in the topology of $\mathfrak{D}_{0}$. A similar statement holds if $\Phi(0) \in \mathfrak{D}$. In either case

$$
\psi(t)=\int A^{ \pm}(k, t) v(k) d k \Phi(t)
$$

is a solution of (7.5). [This follows formally from (7.4).] Thus $A^{ \pm}(k, t)$ is an operator on the Cauchy data of $\Phi$ for the time $t$ which creates or annihilates a particle of momentum $k$ at the time $t=0$. Similarly $A^{ \pm}(k, t-s)$ acts on the same Cauchy data (at time $t$ ) and creates or annihilates a particle at the time $t=s$.

We use the symbol $\mathfrak{w}_{1}$, etc. to denote the class of operators (7.3) which for each fixed $t$ belong to the class $\mathfrak{w}_{1}$, etc. considered earlier.

Lemma 7.1. If $H \in \mathfrak{w}\left(\operatorname{resp} . \mathfrak{w}_{0}, \mathfrak{w}_{1}\right)$ then $(\partial / \partial t) H$ exists in the topology of $\mathfrak{w}$ (resp. $\left.\mathfrak{w}_{0}, \mathfrak{w}_{1}\right)$ and

$$
i \frac{\partial}{\partial t} H=\left[H_{0}, H\right] \text {. }
$$

If $\Phi(0) \in \mathfrak{S}_{0}\left(\right.$ resp. $\left.\mathfrak{S}_{0}, \mathfrak{S}\right)$ then $H \Phi(t)$ is a solution of (7.5) in $\mathfrak{D}$ (resp. $\left.\mathfrak{S}_{0}, \mathfrak{S}\right)$.

Proof. It is clear that the derivative exists and that it defines a derivation of $\mathfrak{w}_{0}=\left\{\lambda H_{0}\right\}+\mathfrak{u}_{0}$ which is continuous in the $\mathfrak{w}$ topology. The right member of (7.6) defines a derivation of $\mathfrak{w}_{0}$ with the same properties. The exponential factors cancel in

$$
H_{0}(t)=\int A^{+}(k, t) \omega(k) A^{-}(k, t) d k,
$$

so $H_{0}(t)=H_{0}(0)$ and (7.6) holds for $H=H_{0}$. For $H=V_{01}$ or $H=V_{10}$, (7.6) is equivalent to (7.4). For a general $H,(7.6)$ follows from these two cases and the derivation property. The last statement follows from (7.6).

Let $Q$ be in $\mathfrak{u}_{1,0}$ and define $B^{ \pm}$by (5.3). We regard the $A^{ \pm}$as independent variables and we regard an $H$ in $\mathfrak{w}_{1}$ as a function of these variables,

$$
H=H\left(A^{ \pm}\right) \text {. }
$$


We use (5.4) to define the operation of "substituting" $B^{ \pm}$for $A^{ \pm}$, and thus we define

$$
H\left(B^{ \pm}\right)=\exp (Q) H\left(A^{ \pm}\right) \exp (-Q) .
$$

If the multiplications in the right member of (7.7) are performed, we obtain a new function of the $A^{ \pm}$

and so

$$
\exp (Q) H\left(A^{ \pm}\right) \exp (-Q)=\tilde{H}\left(A^{ \pm}\right),
$$

$$
H\left(B^{ \pm}\right)=\tilde{H}\left(A^{ \pm}\right) .
$$

These considerations and Lemma 7.1 provide the proof for the following theorem.

Theorem 7.2. Let $Q$ be in $\mathfrak{u}_{1,0}$ and let

Then

$$
\begin{aligned}
H & =\exp (-Q) H_{0} \exp (Q) \\
B^{ \pm} & =\exp (Q) A^{ \pm} \exp (-Q) .
\end{aligned}
$$

$$
i \frac{\partial}{\partial t} B^{ \pm}(k, t)=\left[H\left(B^{ \pm}\right), B^{ \pm}(k, t)\right] .
$$

Since $H\left(B^{ \pm}\right)=H_{0}\left(A^{ \pm}\right)=H_{0}$, we see that (7.5) is equivalent to the Schrödinger equation for a problem with interaction:

$$
i \frac{\partial}{\partial t} \Phi(t)=H\left(B^{ \pm}(0)\right) \Phi(t) .
$$

Let $\Phi \in \mathfrak{D}$ be a solution of (7.8) and let $V \in \mathfrak{u}_{1}$. Then $V\left(B^{ \pm}\right)=\widetilde{V}\left(A^{ \pm}\right)$ with $\widetilde{V} \in \mathfrak{u}_{1}$. Thus

$$
V\left(B^{ \pm}(t)\right) \Phi(t)
$$

is a solution of (7.5) and (7.8).

Next we consider the Hamiltonian

$$
H_{1}=H_{0}+V_{0 r} \text {. }
$$

As above and as in $\S 4$ we define

Then

$$
B^{ \pm}=\exp \left(\Gamma V_{0 r}\right) A^{ \pm} \exp \left(-\Gamma V_{0 r}\right) \text {. }
$$

$$
\begin{aligned}
& B^{-}=A^{-} \\
& B^{+}=A^{+}+\left[\Gamma V, A^{+}\right] .
\end{aligned}
$$

One can show directly that

$$
\begin{aligned}
H_{1}\left(B^{ \pm}\right) & =H_{0}\left(A^{ \pm}\right) \\
i \frac{\partial}{\partial t} B^{ \pm}(t) & =\left[H_{1}\left(B^{ \pm}\right), B^{ \pm}(t)\right] .
\end{aligned}
$$

Finally we consider the Hamiltonian $H$ given by (6.1), (6.2) with $\lambda=1$. Let $Q=Q\left(A^{ \pm}\right)$be given by Theorem 6.1. In a formal sense

$$
C^{ \pm}(t)=\exp \left(Q\left(B^{ \pm}(t)\right)\right) B^{ \pm}(t) \exp \left(-Q\left(B^{ \pm}(t)\right)\right)_{20^{*}}
$$


is a solution of the equation

$$
i \frac{\partial}{\partial t} C^{ \pm}(t)=\left[H\left(C^{ \pm}\right), C^{ \pm}(t)\right]
$$

Although $Q$ and $\exp \left(V_{0 r}\right)$ have meaning as operators on certain Fock type spaces, the space for $Q$ is different than the space for $\exp \left(V_{0 r}\right)$ and the expression

$$
Q\left(B^{ \pm}\right)=\exp \left(\Gamma V_{0 r}\right) Q \exp \left(-\Gamma V_{0 r}\right)
$$

appears not to have a meaning as an operator on the domains we have considered. Consequently we have not found canonical annihilation creation operators $C^{ \pm}(t)$ for particles $H$ by the method of Theorem 7.2.

We continue to consider the same Hamiltonian $H$, but we now do this in the Schrödinger picture. We let $A^{ \pm}$denote time independent annihilation creation operators, as in the previous sections.

Theorem 7.3. Let $H$ and $Q$ be as in Theorem 6.1. Let

Then $T \in \mathfrak{u} \subset L\left(\mathfrak{D}_{0}, \mathfrak{D}\right)$ and

$$
T=\exp (-Q) \exp \left(-\Gamma V_{0 r}\right) \text {. }
$$

$$
H T=T H_{0} .
$$

If $\Phi=\Phi(t)$ is a solution of the free Schrödinger equation (7.5) and if $\Phi(0) \in \mathfrak{S}_{0}$ then

$$
\psi(t)=T \Phi(t)
$$

is a solution of the interacting Schrödinger equation

$$
i \frac{\partial}{\partial t} \psi=H \psi \text {. }
$$

The $Q$ of Theorem 6.1 is not unique. Let

Then

$$
\exp \left(Q_{i}\right) H \exp \left(-Q_{i}\right)=H_{1}, \quad i=1,2 .
$$

and by Theorem 5.7,

$$
\exp \left(-Q_{1}\right) \exp \left(Q_{2}\right) \in I+\mathfrak{c}_{H}
$$

$$
\exp \left(-Q_{1}\right)=\exp (R) \exp \left(-Q_{2}\right)
$$

where $R \in \mathfrak{c}_{H}$. Thus the $T$ in (7.11) is determined up to multiplication on the left by an element of $I+c_{H}$. This nonuniqueness of $T$ does not affect the solubility of (7.13).

Proposition 7.4. Let $\psi(t)$ be a differentiable function of $t$ with values in $\mathfrak{S}$ and let $P$ be in $I+\mathfrak{c}_{H}$. Then $\psi$ is a solution of (7.13) if and only if $P \psi$ is.

Proof of Theorem 7.3. It is evident that the product $T$ in (7.11) exists and defines an element of $\mathfrak{u}$. If $\Phi(0) \in \mathfrak{S}_{0}$ then $\Phi(t) \in \mathfrak{S}_{0}$ and $(\partial / \partial t) \Phi(t)$ exists in $\mathfrak{D}_{0}$. Thus $T \Phi=\psi$ is defined and $(\partial / \partial t) \psi$ exists in $\mathfrak{D}$. Furthermore

$$
\begin{aligned}
H T & =\exp (-Q) H_{1} \exp \left(-\Gamma V_{0 r}\right) \\
& =T H_{0},
\end{aligned}
$$

and this implies that $\psi$ is a solution of (7.12). 
We do not know what physical significance, if any, these solutions have. In $\S 9$ we find solutions which appear to correspond to the standard formal solutions of perturbation theory.

\section{§ 8. The operation $\boldsymbol{\Gamma}$}

The operator $\left[H_{0}, V_{l m}\right]$ has a kernel

where

$$
\left(\sum \omega_{i}-\sum \omega_{j}^{\prime}\right) v_{l m}\left(k, k^{\prime}\right)
$$

$$
\omega_{j}=\omega\left(k_{j}\right), \quad \omega_{j}^{\prime}=\omega\left(k_{j}^{\prime}\right) .
$$

Thus any solution $\Gamma V_{l m}$ of the equation

$$
\left[H_{0}, \Gamma V_{l m}\right]=V_{l m}
$$

will in general have a singular kernel. In this section we derive some properties of such singular operators, following the ideas of [2].

We let $\Omega_{j}$ denote the angular variables $k_{j}|| k_{j} \mid$ and we write

Let

$$
d k_{j}=\omega_{j}\left|k_{j}\right| d \omega_{j} d \Omega_{j} .
$$

$$
\begin{gathered}
\hat{\varphi}(\lambda, \Omega)=\int e^{-i \lambda \cdot \omega} \varphi(k) d \omega \\
\hat{v}\left(\lambda, \lambda^{\prime}, \Omega, \Omega^{\prime}\right)=\int e^{-i\left(\lambda \cdot \omega-\lambda^{\prime} \omega^{\prime}\right)} v\left(k, k^{\prime}\right) d \omega d \omega^{\prime} .
\end{gathered}
$$

Actually it is not these Fourier transforms which interest us, but rather the transform in the next lemma.

Lemma 8.1. Let $\varphi \in \mathscr{S}$. There is a constant $M$ such that

$$
\left|\left[\Pi_{j}\left(\omega_{j}\left|k_{j}\right|\right)^{1 / 2} \varphi\right]^{\wedge}(\lambda)\right| \leqq M \Pi_{j}\left(1+\left|\lambda_{j}\right|\right)^{-5 / 4},
$$

and similarly for $v$.

Proof. (Cf. [4], p. 124.) It is sufficient to consider the case where $\varphi=\varphi_{1}$ depends on $k_{1}$ only. We write

Then (8.2) is given by

$$
\begin{aligned}
|k| & =\left(\omega^{2}-\mu^{2}\right)^{1 / 2} \\
& =(\omega-\mu)^{1 / 2}(\omega+\mu)^{1 / 2} .
\end{aligned}
$$

$$
\left|\int_{\mu}^{\infty} e^{-i \lambda \omega}(\omega-\mu)^{1 / 4} \chi\left((\omega-\mu)^{1 / 2}\right) d \omega\right|=\left|2 \int_{0}^{\infty} e^{-i \lambda \tau^{2}} \tau^{3 / 2} \chi(\tau) d \tau\right|,
$$

where $\chi(\tau)=\left(\tau^{2}+\mu\right)^{1 / 2}\left(\tau^{2}+2 \mu\right)^{1 / 2} \varphi\left(\tau\left(\tau^{2}+2 \mu\right)^{1 / 2} \Omega\right)$ is in $\mathscr{S}$. We consider first the case $\chi_{1}=a \exp \left(-\tau^{2}\right)$. If we substutite this in (8.3) and deform the contour of integration in the complex plane, we obtain

$$
2 a \int_{0}^{\infty} \exp \left(-|1+i \lambda| \tau^{2}\right) \tau^{3 / 2} d \tau \leqq 2 a(1+|\lambda|)^{-5 / 4} \int_{0}^{\infty} e^{-\tau^{2}} \tau^{3 / 2} d \tau .
$$


Next we consider the case $\chi_{2}=b \tau \exp \left(-\tau^{2}\right)$. As before we obtain in this case the bound

$$
2 b(1+|\lambda|)^{-7 / 4} \int_{0}^{\infty} e^{-\tau^{2}} \tau^{5 / 2} d \tau
$$

for (8.3). We set $a=\chi(0), b=\chi^{\prime}(0)$ and

Then

$$
\chi_{3}=\chi-\chi_{1}-\chi_{2} .
$$

$$
(\omega-\mu)^{1 / 4} \chi_{3}\left((\omega-\mu)^{1 / 2}\right)=2^{-1} \chi_{3}^{\prime \prime}(0)(\omega-\mu)^{5 / 4}+\cdots
$$

has two derivatives in $L_{1}$. Thus

$$
\left|\int_{\mu}^{\infty} e^{-i \lambda \omega}(\omega-\mu)^{1 / 4} \chi_{3}\left((\omega-\mu)^{1 / 2}\right) d \omega\right| \leqq \text { const. }(1+|\lambda|)^{-2} .
$$

The lemma follows from this, (8.4) and (8.5).

We now define

$$
F \varphi=\left[\Pi_{j}\left(\omega_{j}\left|k_{j}\right|\right)^{1 / 2} \varphi\right]^{\wedge},
$$

and similarly for $v$. Let $\Lambda_{j}=i \partial / \partial \lambda_{j}$, let $\nu=v_{1}, \ldots, v_{n}$ be a multiindex and let

$$
\Lambda^{v}=\Pi_{j} \Lambda_{j}^{v_{j}}
$$

If $\varphi=\varphi_{0}$, define $\|\varphi\|_{\nu}=|\varphi|$. Now suppose $\varphi=\varphi_{n}(k)$ where $k$ has $3 n$ components. Let $\tau$ be a vector with $r$ components (each a real number) and with $\tau_{1}=0$ and let $j$ be a function,

Define

$$
j:\{1, \ldots, n\} \rightarrow\{1, \ldots, r\} .
$$

$$
\begin{gathered}
\lambda+\tau=\lambda_{1}+\tau_{j(1)}, \ldots, \lambda_{n}+\tau_{j(n)} \\
d \tau=d \tau_{2} \ldots d \tau_{r} \\
\|\varphi\|_{j, v}=\sup _{\lambda, \Omega} \int\left|A^{v} F \varphi(\lambda+\tau, \Omega)\right| d \tau \\
\|\varphi\|_{v}=\sup _{j}\|\varphi\|_{j, v} .
\end{gathered}
$$

Let $L^{\wedge}$ be the completion of $\mathscr{S}$ in the set of seminorms (8.7). Each $\varphi$ in $L^{\wedge}$ is a rapidly decreasing continuous function. If $\sigma$ is a real number and $\lambda+\sigma=\lambda_{1}+\sigma, \ldots, \lambda_{n}+\sigma$ we define

$$
\gamma \varphi=-i F^{-1} \int_{\sigma \leqq 0} F \varphi(\lambda+\sigma) d \sigma .
$$

[We get this definition of $\lambda+\sigma$ if $j(i) \equiv 2=r$ in (8.6).] The inverse Fourier transformation in (8.8) is taken in the sense of distributions. These definitions apply also with kernels $v_{l m}$ replacing the function $\varphi_{n}$. Let

$$
(k)_{n}=k_{1}, \ldots, k_{n}
$$


denote a vector with $3 n$ components and let

$$
\left\langle\varphi_{n}, \psi_{m}\right\rangle_{s}=\int \overline{\varphi_{n}}\left(\left(k^{\prime}\right)_{n-s},(k)_{s}\right) \psi_{m}\left(\left(k^{\prime \prime}\right)_{m-s},(k)_{s}\right) d k
$$

if $n, m \geqq s$ and let $\left\langle\varphi_{n}, \psi_{m}\right\rangle_{s}=0$ otherwise.

Lemma 8.2. Let $\varphi_{n}$ and $\psi_{m}$ be in $L^{\wedge}$. Then

$$
\left\|\left\langle\varphi_{n}, \psi_{m}\right\rangle_{s}\right\|_{v} \leqq K^{s}\left\|\varphi_{n}\right\|_{\nu}\left\|\psi_{m}\right\|_{\nu} \text {. }
$$

If $s \geqq 1$ then

$$
\begin{aligned}
& \left\|\left\langle\gamma \varphi_{n}, \psi_{m}\right\rangle_{s}\right\|_{v} \leqq K^{s}\left\|\varphi_{n}\right\|_{v}\left\|\psi_{m}\right\|_{v} \\
& \left\|\left\langle\varphi_{n}, \gamma \psi_{m}\right\rangle_{s}\right\|_{v} \leqq K^{s}\left\|\varphi_{n}\right\|_{v}\left\|\psi_{m}\right\|_{\nu} .
\end{aligned}
$$

Proof. We use the Fourier transform to express the integration in (8.9) as integrals with respect to $\lambda$ and $\Omega$. The operator $\Lambda^{v}$ does not effect the proof and so we only consider the case $\nu=0$. Let a function $j$ be given. If an integration occurs with respect to some variable $\tau_{i}$ which affects $\varphi$ but not $\psi$, we have

$$
\int|\varphi \psi| d \tau_{i}=|\psi| \int|\psi| d \tau_{i}
$$

If $\tau_{i}$ affects both variables we use the bound

We find

$$
\left.\int|\varphi \psi| d \tau_{i}=\sup _{\tau_{i}}|\varphi|\right) \int|\psi| d \tau_{i}
$$

$$
\begin{aligned}
\left\|\langle\varphi, \psi\rangle_{s}\right\|_{j} & =(2 \pi)^{r} \sup \int\left|\bar{F} \varphi\left(\lambda^{\prime}+\tau, \lambda\right) F \psi\left(\lambda^{\prime \prime}+\tau, \lambda\right)\right| d \tau d \lambda d \Omega \\
& \leqq(2 \pi)^{r}\left(\int d \Omega\right) \sup \int\left|\bar{F} \varphi\left(\lambda^{\prime}+\tau, \lambda\right) F \psi\left(\lambda^{\prime \prime}+\tau, \lambda\right)\right| d \tau d \lambda .
\end{aligned}
$$

We write

$$
\tau=\varrho_{1}+\varrho_{2}
$$

where $\varrho_{1}$ is the part of $\tau$ which affects the variables of $\varphi$ alone. Then

$$
\begin{aligned}
\int \mid F \varphi\left(\lambda^{\prime}+\tau, \lambda\right) F & \psi\left(\lambda^{\prime \prime}+\tau, \lambda\right) \mid d \tau d \lambda \\
= & \int\left[\int\left|F \varphi\left(\lambda^{\prime}+\varrho_{1}+\varrho_{2}, \lambda\right)\right| d \varrho_{1}\right]\left|F \psi\left(\lambda^{\prime \prime}+\varrho_{2}, \lambda\right)\right| d \varrho_{2} d \lambda \\
& \leqq\left[\sup _{\lambda^{\prime}, \lambda} \int \mid F \varphi\left(\lambda^{\prime}+\varrho_{1}, \lambda\right) d \varrho_{1}\right] \int\left|F \psi\left(\lambda^{\prime \prime}+\varrho_{2}, \lambda\right)\right| d \varrho_{2} d \lambda \\
& \leqq\|\varphi\|_{j_{*}}\|\psi\|_{j}
\end{aligned}
$$

for some new function $j_{*}$. Thus (8.10) holds with $K=2 \pi \int d \Omega_{1}=8 \pi^{2}$.

We now suppose $s \geqq 1$ and let $\sigma$ be a real number.

$$
\begin{aligned}
\left\|\langle\gamma \varphi, \psi\rangle_{s}\right\|_{j} & \leqq K^{s} \sup \int\left|F \gamma \varphi\left(\lambda^{\prime}+\varrho_{1}, \lambda\right)\right| d \varrho_{1}\|\psi\|_{j} \\
& \leqq K^{s}\|\psi\|_{j} \sup \iint\left|F \varphi\left(\lambda^{\prime}+\varrho_{1}+\sigma, \lambda+\sigma\right)\right| d \sigma d \varrho_{1} .
\end{aligned}
$$

We substitute $\varrho_{1}-\sigma$ for $\varrho_{1}$, which eliminates $\sigma$ from the variables affected by $\varrho_{1}$ and gives us

$$
\left\|\langle\gamma \varphi, \psi\rangle_{s}\right\|_{j}=K^{s}\|\psi\|_{j}\|\varphi\|_{j_{* *}}
$$


for some new function $j_{* *}$. This proves (8.11). The proof of (8.12) is similar but requires reversing the roles of $\varphi$ and $\psi$ in (8.13).

This lemma applies with $\varphi$ and/or $\psi$ replaced by kernels $v_{i j}$ and/or $v_{l m}$. Thus the $v$ norm of any of the following products,

$$
V_{i j} V_{l m},\left[\Gamma V_{i j}, V_{l m}\right], V_{l m} \varphi_{n}
$$

or

$$
\left.\Gamma V_{l m} \varphi_{n} \quad \text { (provided } m>0\right),
$$

is bounded in terms of a product

$$
\left\|v_{i j}\right\|_{\nu}\left\|v_{l m}\right\|_{\nu} \text { or }\left\|v_{l m}\right\|_{\nu}\left\|\varphi_{n}\right\|_{\nu} .
$$

The restriction $s \geqq 1$ in (8.11) and (8.12) interferes with the products

$$
\Gamma V_{l 0} \varphi_{n},\left(\Gamma V_{i j}\right) V_{l m},:\left(\Gamma V_{i j}\right) V_{l m}: \text {. }
$$

The next lemma yields some continuity for $\Gamma V_{l_{0}} \varphi_{n}$ provided $v_{l_{0}} \in \mathscr{S}$, since it shows that in this case $\gamma v_{l 0} \in \mathscr{S} \subset L^{\wedge}$.

Lemma 8.3. Let $v_{l 0}$ and $v_{0 m}$ be in $L^{\wedge}, l \neq 0 \neq m$. Then

$$
\begin{aligned}
\gamma v_{l 0}(k) & =\left(\sum \omega_{j}\right)^{-1} v_{l 0}(k) \\
\gamma v_{0 m}(k) & =-\left(\sum \omega_{j}\right)^{-1} v_{0 m}(k) .
\end{aligned}
$$

Proof. Let $\varphi$ be in $\mathscr{S}$. Then for $v=v_{l 0}$, $\langle\varphi, \gamma v\rangle=$ const. $\langle F \varphi, F \gamma v\rangle=-$ const. $i \int_{\sigma \leqq 0} \bar{F} \varphi(\lambda) F v(\lambda+\sigma) d \lambda d \sigma$, since the integrand is in $L_{1}$. Thus

$$
\begin{aligned}
\langle\varphi, \gamma v\rangle= & -\lim _{N \rightarrow \infty} \int_{-N}^{0}\left\langle\varphi, i e^{-i(\Sigma \omega) \sigma} v\right\rangle d \sigma \\
= & \lim _{N \rightarrow \infty} \int_{-N}^{0}(d / d \sigma)\left\langle\varphi,\left(\sum \omega\right)^{-1} e^{-i(\Sigma \omega) \sigma} v\right\rangle d \sigma \\
= & \left\langle\varphi,\left(\sum \omega\right)^{-1} v\right\rangle-\lim _{N \rightarrow \infty} \int \bar{\varphi}\left(\sum \omega\right)^{-1} e^{i(\Sigma \omega) N} v d k \\
= & \left\langle\varphi,\left(\sum \omega\right)^{-1} v\right\rangle-\lim _{N \rightarrow \infty} \int \bar{\varphi}\left(\sum \omega\right)^{-1} e^{i(\Sigma \omega) N} \times \\
& \times v \prod_{j}\left(k_{j} \omega_{j}\right)^{1 / 2} d \Omega d \omega_{1} \ldots d \omega_{l-1} d(\Sigma \cdot \omega) \\
= & \left\langle\varphi,\left(\sum \omega\right)^{-1} v\right\rangle
\end{aligned}
$$

by the Riemann Lebesgue lemma.

We set $\mathfrak{S}_{0}=\mathfrak{S}_{0}(\mathscr{S})$ and $\mathfrak{D}=\mathfrak{D}(\mathscr{S})$. Let $\mathfrak{D}\left(L^{\wedge}\right)$ be defined as the space of sequences

$$
\Phi=\left\{\varphi_{0}, \varphi_{1}, \ldots\right\}
$$

for which $\varphi_{j}$ is a symmetric function of the variables $k_{1}, \ldots, k_{j}$ and $\varphi_{j} \in L^{\wedge}$. Let $\mathfrak{D}_{0}\left(L^{\wedge}\right)$ be the subspace consisting of those $\Phi$ for which $\varphi_{j}=0$ for all large $j$. We set $\mathfrak{u}_{1}=\mathfrak{u}_{1}(\mathscr{S})$, etc. and we define $\mathfrak{u}_{1}\left(L^{\wedge}\right)$, etc. in a similar fashion. 
If $V \in \mathfrak{u}\left(L^{\wedge}\right)$ and if $V=\sum_{(l, m) \neq 0,0} V_{l m}$, we define

$$
\Gamma V=\sum \Gamma V_{l m}
$$

where $\Gamma V_{l m}$ is defined as an operator with kernel $\gamma v_{l m}$. We define the seminorms

$$
\begin{aligned}
\|\Phi\|_{v, n} & =\sup _{j \leqq n}\left\|\varphi_{j}\right\|_{v}, \Phi \in \mathfrak{O}\left(L^{\wedge}\right) \\
\|V\|_{v, n} & =\sup _{l \leqq n}\left\|v_{l m}\right\|_{v}, V \in \mathfrak{u}_{1, r}\left(L^{\wedge}\right) .
\end{aligned}
$$

These seminorms define a topology in $\mathfrak{S}\left(L^{\wedge}\right), \mathfrak{u}_{1, r}\left(L^{\wedge}\right)$ and

We give

$$
\mathfrak{w}_{1, r}\left(L^{\wedge}\right)=\left\{\lambda H_{0}\right\} \oplus \mathfrak{u}_{1, r}\left(L^{\wedge}\right) \text {. }
$$

$$
\mathfrak{w}_{1}\left(L^{\wedge}\right)=\cup_{r} \mathfrak{w}_{1, r}\left(L^{\wedge}\right)
$$

the inductive limit topology.

Proposition 8.4. Let $V_{1} \in \mathfrak{u}_{1,-r}\left(L^{\wedge}\right)$ and $V_{2} \in \mathfrak{u}_{1,-s}\left(L^{\wedge}\right)$. Suppose $\left(V_{1}\right)_{00}=0$. Then

$$
\begin{array}{r}
\left\|V_{1} \Phi\right\|_{v, n} \leqq \text { const. }\left\|V_{1}\right\|_{v, n}\|\Phi\|_{v, n+r} \\
\left\|V_{1} V_{2}\right\|_{v, n} \leqq \text { const. }\left\|V_{1}\right\|_{v, n}\left\|V_{2}\right\|_{v, n+r}, r \geqq 0 \\
\left\|\left[\Gamma V_{1}, V_{2}\right]\right\|_{\nu, n} \leqq \text { const. }\left\|V_{1}\right\|_{v, n}\left\|V_{2}\right\|_{v, n+r}, r \geqq 0 .
\end{array}
$$

If $\left(V_{1}\right)_{l_{0}}=0$ then

$$
\left\|\Gamma V_{1} \Phi\right\|_{\nu, n} \leqq \text { const. }\left\|V_{1}\right\|_{\nu, n}\|\Phi\|_{\nu, n+r} .
$$

The constant depends only on $n, r$ and $s$.

Proof. This follows from Lemma 8.2 together with the observation that the left hand sides of these inequalities depend only on certain low order terms of $V_{1}, V_{2}$ and $\Phi$. (Cf. Lemma 3.3.)

We consider the elements of $\mathfrak{w}_{1}\left(L^{\wedge}\right)$ as operators on $\mathfrak{S}\left(L^{\wedge}\right) \cdot \mathfrak{w}_{1}\left(L^{\wedge}\right)$ is a Lie algebra, $\mathfrak{u}_{1}\left(L^{\wedge}\right)$ is an algebra and

$$
\left[\Gamma V_{1}, V_{2}\right] \in \mathfrak{u}_{1}\left(L^{\wedge}\right)
$$

if $V_{i} \in \mathfrak{u}_{1}\left(L^{\wedge}\right)$ and $\left(V_{1}\right)_{l_{0}}=0$. (If $\left(V_{1}\right)_{00}=0$ but $\left(V_{1}\right)_{l 0} \neq 0$ for some $l$ then the commutator is formally an element of $\mathfrak{u}_{1}\left(L^{\wedge}\right)$ but might not be everywhere defined.)

Theorem 8.5. Let

$$
P=Q_{1}+\Gamma Q_{2}
$$

be given with $Q_{i} \in \mathfrak{u}_{1,0}\left(L^{\wedge}\right)$ and $\left(Q_{2}\right)_{l 0}=0$. Then the power series for $\exp P$ and $\exp (\operatorname{ad} P)$ converge uniformly on bounded sets in $\mathfrak{S}\left(L^{\wedge}\right)$ and in $\mathfrak{w}_{1}\left(L^{\wedge}\right)$ respectively. If $H \in \mathfrak{w}_{1}\left(L^{\wedge}\right)$ then

$$
\exp (P) H \exp (-P)=\exp (\operatorname{ad} P) H \text {. }
$$

Proof. The inequalities in Proposition 8.4 show that there is uniform convergence with respect to each seminorm. 
Theorem 8.6. Let $V \in \mathfrak{u}_{1}\left(L^{\wedge}\right)$ and let $v_{l_{0}} \in \mathscr{S}$ and $V_{00}=0$. Then

Proof. We have

$$
\left[H_{0}, \Gamma V\right]=V \text {. }
$$

$$
F H_{0} \varphi_{n}=F\left(\sum_{i=1}^{n} \omega_{j}\right) \varphi_{n}=\sum \Lambda_{j} F \varphi_{n}
$$

and for some constant $\alpha=\alpha(l, m, n)$ we have

$$
F V_{l m} \varphi_{n}=\alpha \operatorname{Sym} \int F v_{l m}\left(\lambda^{\prime}, \lambda^{\prime \prime}\right) F \varphi_{n}\left(\lambda^{\prime \prime},(\lambda)_{n-m}\right) d \lambda^{\prime \prime} d \Omega^{\prime \prime} .
$$

After integrating by parts and cancelling terms we find

$$
\begin{aligned}
F\left[H_{0}, \Gamma V_{l m}\right] \varphi_{n} & =-i \alpha \operatorname{Sym} \iint_{\sigma \leqq 0}\left[\left(\sum_{j=1}^{l} \Lambda_{j}^{\prime}+\sum_{j=1}^{m} \Lambda_{j}^{\prime \prime}\right) F v_{l m}\left(\lambda^{\prime}+\sigma, \lambda^{\prime \prime}+\sigma\right)\right] \\
& F \varphi_{n}\left(\lambda^{\prime \prime},(\lambda)_{n-m}\right) d \sigma d \lambda^{\prime \prime} d \Omega^{\prime \prime} \\
= & \alpha \operatorname{Sym} \iint_{\sigma \leqq 0}(d / d \sigma) F v_{l m}\left(\lambda^{\prime}+\sigma, \lambda^{\prime \prime}+\sigma\right) F \varphi_{n}\left(\lambda^{\prime \prime},(\lambda)_{n-m}\right) d \sigma d \lambda^{\prime \prime} d \Omega^{\prime \prime} \\
= & F V_{l m} \varphi_{n} .
\end{aligned}
$$

§9. Solutions of the Schrödinger equation which have a partial perturbation expansion

We now consider a Hamiltonian of the form

$$
\begin{aligned}
& H=H_{0}+\varepsilon V \in \mathfrak{w}_{1}(\mathscr{S}) \\
& V=\sum_{l-m>-r} V_{l m}+V_{0 r}
\end{aligned}
$$

where $\varepsilon$ is a parameter, $r>0$ and $V_{0 r} \neq 0$. Let

$$
H_{\text {ren }}=H-E
$$

denote the renormalized Hamiltonian. $E$ is a (finite) multiple of the identity operator. In this section we find a $T$ which intertwines $H_{\text {ren }}$ and $H_{0}$,

$$
H_{\text {ren }} T=T H_{0},
$$

and the "low order" parts of $T$ depend analytically on $\varepsilon$.

Theorem 9.1. Let $V, \varepsilon$ and a positive integer $n$ be given. There is a renormalization constant $E$ and a continuous transformation $T$ from $D_{0}\left(L^{\wedge}\right)$ into $D\left(L^{\wedge}\right)$ which solve (9.4). If

$$
\Psi=T \Phi=T\left\{\varphi_{0}, \ldots, \varphi_{j}, 0, \ldots\right\}
$$

is in the range of $T$ then the terms

depend analytically on $\varepsilon$.

$$
\psi_{0}, \ldots, \psi_{n}
$$

As a consequence of this theorem,

$$
\Psi(t, \varepsilon)=T(\varepsilon) \exp \left(-i t H_{0}\right) \Phi(0)
$$

is a solution of the renormalized Schrödinger equation

$$
i \frac{\partial}{\partial t} \Psi=H_{\mathrm{ren}} \Psi
$$


in the space $\mathfrak{S}\left(L^{\wedge}\right)$, and the low order terms (9.5) of $\Psi$ depend analytically on $\varepsilon$. We conjecture that these terms agree with standard perturbation theory up to any desired finite order (for example, $n-1$ ) if $T$ is suitably chosen.

Lemma 9.2. There are polynomials

$$
Q(\varepsilon)=\varepsilon Q^{(1)}+\cdots+\varepsilon^{2 n} Q^{(2 n)}
$$

and

$$
R(\varepsilon)=\varepsilon R^{(1)}+\cdots+\varepsilon^{2 n} R^{(2 n)}
$$

with coefficients

and

$$
Q^{(j)}=\sum_{l>0} Q_{l 0}^{(j)} \in \mathfrak{u}_{1}(\mathscr{S})
$$

$$
R^{(j)}=\sum_{l \geqq m>0} R_{l m}^{(j)} \in \mathfrak{u}_{1}\left(L^{\wedge}\right)
$$

and there is a constant $E$ and there is an $H^{\prime \prime}$ in $\mathfrak{w}_{1}\left(L^{\wedge}\right)$; for these operators the relation

$$
\exp (\Gamma R) \exp (\Gamma Q) H_{\text {ren }} \exp (-\Gamma Q) \exp (-\Gamma R)=H^{\prime \prime}
$$

is valid. Furthermore they can be chosen so that

$$
\begin{aligned}
& \left(H^{\prime \prime}-H_{0}\right)_{l m}=0 \text {, } \\
& l-m<-r \text { or } l=m=0 \\
& \left(H^{\prime \prime}-H_{0}\right)_{l m}=\varepsilon W_{l m}^{(1)}+\cdots, \quad-r \leqq l-m<0 \\
& \left.\left(H^{\prime \prime}-H_{0}\right)_{l m}=\varepsilon^{2 n+1} W_{l m}^{(2 n)}\right)+\cdots, \quad 0 \leqq l-m, l \neq 0 \text {. }
\end{aligned}
$$

The $+\cdots$ signify higher order terms in a convergent series.

Proof. Let $Q^{(0)}=0$ and $Q_{j}=\Sigma_{0 \leqq i \leqq j} \varepsilon^{i} Q^{(i)}$. We proceed by induction and so we may suppose $Q_{j}$ defined for some $j \geqq 0$. Let

We suppose

$$
\exp \left(\Gamma Q_{j}\right) H \exp \left(-\Gamma Q_{j}\right)=H^{\prime}(j)
$$

$$
\left(H^{\prime}(j)-H_{0}\right)_{l_{0}}=\varepsilon^{j+1} Y_{l 0}^{(j+1)}+\cdots, \quad l>0
$$

and we suppose $H^{\prime}(j) \in \mathfrak{w}_{1}(\mathscr{S})$. Let

Then

$$
Q^{(j+1)}=\sum_{0<l} Y_{l 0}^{(j+1)} .
$$

$$
\begin{aligned}
H^{\prime}(j+1) & =\exp \left(\Gamma Q_{j+1}\right) H \exp \left(-\Gamma Q_{j+1}\right) \\
& =\exp \left(\Gamma Q_{j}\right) H \exp \left(-\Gamma Q_{j}\right)+\sum_{0<l} \varepsilon^{j+1}\left[\Gamma Y^{(j+1)}, H_{0}\right] \\
& =H^{\prime}(j)-\varepsilon^{j+1} Q^{(j+1)} .
\end{aligned}
$$

This proves (9.11) for $j+1$. By the definition (9.12), $Q^{(j+1)} \in \mathfrak{u}_{1}(\mathscr{S})$. By Lemma 8.3, $\Gamma Q^{(j+1)} \in \mathfrak{u}_{1}(\mathscr{S})$ and by Theorem $5.5, H^{\prime}(j+1) \in \mathfrak{w}_{1}(\mathscr{S})$. This completes the inductive definition of $Q$. Let $H^{\prime}=H(2 n)$. Then

$$
\exp (\Gamma Q) H \exp (-\Gamma Q)=H^{\prime} \text {. }
$$


Let $R^{(0)}=0$ and let $R_{1}=\sum_{0 \leqq i \leqq j} \varepsilon^{i} R^{(i)}$. We use a similar induction; let

Then

$$
\exp \left(\Gamma R_{j}\right) H^{\prime} \exp \left(-\Gamma R_{j}\right)=H^{\prime \prime}(j) .
$$

$$
\left(H^{\prime \prime}(j)-H_{0}\right)_{l m}=\varepsilon^{j+1} Z_{l m}^{(j+1)}+\cdots, \quad l \geqq m, l \neq 0
$$

and $\left(H^{\prime \prime}(j)-H_{0}\right)_{l 0}=\varepsilon^{2 n+1} Z_{l 0}^{(2 n+1)}+\cdots$ for $l>0$. Let

Then

$$
\begin{aligned}
R^{(j+1)} & =\sum_{l \geqq m>0} Z_{l m}^{(j+1)} \\
E & =\left(H^{\prime \prime}(2 n)\right)_{00} \\
H^{\prime \prime} & =H^{\prime \prime}(2 n)-E .
\end{aligned}
$$

$$
\exp (\Gamma R)\left(H^{\prime}-E\right) \exp (-\Gamma R)=H^{\prime \prime}
$$

and this combined with (9.13) yields (9.6) and completes the proof.

Let $W^{\prime \prime}$ be the sum of the terms occuring in (9.9) and let

and let

$$
\begin{aligned}
H_{2} & =H^{\prime \prime}-W^{\prime \prime} \\
H_{1} & =\exp (-\Gamma R) \exp (-\Gamma Q) H_{2} \exp (\Gamma Q) \exp (\Gamma R) \\
& =H_{\text {ren }}-\exp (-\Gamma R) \exp (-\Gamma Q) W^{\prime \prime} \exp (\Gamma Q) \exp (\Gamma R) \\
& =H_{\text {ren }}-\exp (-\Gamma R) \exp (-\Gamma Q) W^{\prime \prime} \exp (\Gamma Q) \exp (\Gamma R)
\end{aligned}
$$

$$
W_{1}=-\exp (-\Gamma R) \exp (-\Gamma Q) W^{\prime \prime} \exp (\Gamma Q) \exp (\Gamma R) .
$$

Then $W_{1} \in \mathfrak{u}_{1,0}\left(L^{\wedge}\right)$ and

$$
W_{1}=\varepsilon^{2 n+1} W_{1}^{(2 n+1)}+\cdots
$$

Lemma 9.3. There is a $P=P(\varepsilon)$ in $\mathfrak{u}_{1,1}\left(L^{\wedge}\right)$ such that

$$
\exp (P) H_{\mathrm{ren}} \exp (-P)=H_{1} \text {. }
$$

If $l-m=\mu$ then $P_{l m}$ has an expansion

$$
P_{l m}=\varepsilon^{2 n-\mu} P_{l m}^{(2 n-\mu)}+\cdots
$$

which converges for $\varepsilon \neq 0$ (and for $\varepsilon=0$ if $\mu \leqq 2 n$ ).

Proof. First we discuss the equation

$$
\left(\left[P_{l+r, m}, \varepsilon V_{0 r}\right]\right)_{l m}=F_{l m}
$$

where $F_{l m}$ is given and $P_{l+r, m}$ is the unknown. The proof of Theorem 4.1 gives us

Lemma 9.4. Let $F_{l m} \in \mathfrak{u}_{1,0}\left(L^{\wedge}\right)$ and let $\varepsilon \neq 0$. A solution $P_{l+r, m}$ in $\mathfrak{u}_{1,1}\left(L^{\wedge}\right)$ to $(9.17)$ can be found which is a continuous linear function of $F_{l m}$.

It follows that if

then

$$
F_{l m}=\varepsilon^{j} F_{l m}^{(j)}+\cdots
$$

$$
P_{l+r, m}=\varepsilon^{j-1} P_{l+r, m}^{(j-1)}+\cdots
$$


and (9.19) converges if (9.18) does. We construct $P$ by induction on $\mu$. If $l-m<r$ set $P_{l m}=0$. Suppose

$$
P_{\mu}=\sum_{l-m \leqq \mu} P_{l m}
$$

has been defined so that $(9.16)$ holds and

$$
\left(\exp \left(P_{\mu}\right) H_{\text {ren }} \exp \left(-P_{\mu}\right)\right)_{l m}=\left(H_{1}\right)_{l m}
$$

if $l-m \leqq \mu-r$. (This is true if $\mu=r-1$.) In order to achieve (9.21) for $\mu+1$, we only need

$$
\begin{aligned}
{\left[P_{m+\mu+1, m}, \varepsilon V_{0 r}\right]_{m+\mu+1-r, m} } & \\
& =\left\{-\exp \left(P_{\mu}\right) H_{\text {ren }} \exp \left(-P_{\mu}\right)+H_{\text {ren }}+W_{1}-\right. \\
& \left.-\sum_{j=1}^{r-1}\left[P_{m+\mu+1-j, m-j}, V_{0 r}\right]\right\}_{m+\mu+1-r, m} .
\end{aligned}
$$

This is solved by induction on $m$, using Lemma 9.4 .

To complete the induction on $\mu$ we must verify (9.16) for $\mu+1$. The first two terms in the right member of (9.22) contribute a sum of terms of the form

$$
\text { ad } P_{m_{1}+k_{1}, m_{1}} \ldots \text { ad } P_{m_{j}+k_{j}, m_{j}}\left(H_{\text {ren }}\right)_{m_{0}+\varrho, m_{0}},
$$

where $j \geqq 1,0 \leqq k_{i} \leqq \mu$ and

However, $-\varrho \leqq r$ and so

$$
\sum_{i=1}^{j} k_{i}+\varrho=\mu+1-r .
$$

$$
\sum_{i=1}^{i} k_{i}=\mu+1-(\varrho+r) \leqq \mu+1 .
$$

If $\varrho=-r$ then $\left(H_{\mathrm{ren}}\right)_{m_{0}+\varrho, m_{0}}=\varepsilon V_{0 r}$ if $m_{0}=r$ and is zero otherwise. Thus in this case $H_{\text {ren }}$ contributes a power of $\varepsilon$ to (9.23). If $\varrho>-r$ then the inequality prevails in (9.24). Combining these two cases and using (9.16) we see that the minimum exponent of $\varepsilon$ occuring in (9.23) is at least $2 j n-\mu \leqq 2 n-\mu$. It follows that (9.16) is true for $P_{\mu+1,0}$, and by an induction on $m$, it is true for $P_{m+\mu+1, m}$. This completes the induction on $\mu$. (9.15) follows from (9.21) and the proof is complete.

Let

We now have

$$
T_{1}=\exp (-P) \exp (-\Gamma Q) \exp (-\Gamma R)
$$

$$
H_{\mathrm{ren}} T_{1}=T_{1} H_{2},
$$

or in other words, $H_{\text {ren }}$ has been put in "triangular form".

Lemma 9.5. There is an operator

such that

$$
U=\sum_{l<m} U_{l m}
$$

The terms

$$
\exp (\Gamma U) H_{2} \exp (-\Gamma U)=H_{0}
$$

$$
U_{l m}=\varepsilon U_{l m}^{(1)}+\cdots
$$

are analytic functions of $\varepsilon$ with coefficients $U_{l m}^{(j)}$ in $\mathfrak{u}_{1}\left(L^{\wedge}\right)$. 
We give

$$
\mathfrak{S}_{0}\left(L^{\wedge}\right)=\cup_{k} \mathfrak{D}^{k}\left(L^{\wedge}\right)
$$

the inductive limit topology. Each subspace $\mathfrak{D}^{k}\left(L^{\wedge}\right)$ of states with at most $k$ particles gets its topology from the seminorms (8.14), $v=1,2, \ldots$, $n=k$. We realize expressions of the form (9.25) as operators on $\mathfrak{S}_{0}\left(L^{\wedge}\right)$. We assert that the power series $\exp (\Gamma U)$ converges and that $\Gamma U$ and $\exp \Gamma U$ are continuous operators. It is sufficient to prove this on each subspace $\mathfrak{S}^{k}\left(L^{\wedge}\right)$. However on such a subspace at most $k+1$ terms of the power series are nonzero. Thus the convergence is trivial and continuity follows from Lemma 8.2 (or Proposition 8.4). Let

$$
T=T_{1} \exp (-\Gamma U) \text {. }
$$

Our lemmas show that (9.4) holds, and so Theorem 9.1 follows from Lemma 9.5.

Proof of Lemma 9.5. Let

$$
U_{j}=\Sigma_{l-m=-j} U_{l m} .
$$

Let $U_{j}=0$ for $j \leqq 0$ and by induction on $j$ choose $U_{j}$ so that

$$
\exp \left(\Gamma \sum_{i=1}^{j} U_{i}\right) H_{2} \exp \left(-\Gamma \sum_{i=1}^{j} U_{i}\right)-H_{0}
$$

only contains nonzero terms $W_{l m}(j)$ for which $l+j+1 \leqq m$. We set

$$
U_{j+1}=\sum_{l-m=-(j+1)} W_{l m}(j)
$$

and check that (9.27) has the correct form. Thus $U$ is defined and (9.26) holds. The analyticity of $U$ follows from that of $\mathrm{H}_{2}$ together with the fact that each term $U_{l m}$ of $U$ depends (in a continuous manner) on only a finite number of the terms of $H_{2}-H_{0}$.

\section{Bibliography}

[1] Frredrichs, K. O.: Mathematical aspects of the quantum theory of fields. New York: Interscience 1953.

[2] - Perturbation of spectra in Hilbert space, Lectures in applied mathematics, Vol. III, American Mathematical Society, 1965.

[3] JACOBson, N.: Lie algebras. New York: Interscience 1962.

[4] Jost, R.: The general theory of quantized fields, Lectures in applied mathematics, Vol. IV, American Mathematical Society, 1965.

[5] Kristensen, P., L. Mejlbo, and E. Poulsen: Tempered distributions in infinitely many dimensions I, Comm. Math. Phys. 1, 175-215 (1965).

[6] - - Tempered distributions in infinitely many dimensions II, Math. Scand. 14, 129-150 (1964).

[7] - - Tempered distributions in infinitely many dimensions III, to appear.

[8] Nelson, E.: Interaction of nonrelativistic particles with a scalar field. J. Math. Phys. 5, 1190-1197 (1964).

[9] Wrghtman, A.: Introduction to some aspects of the relativistic dynamics of quantized fields, Institute des Hautes Etudes Scientifiques, Bures-surYvette (Revised notes for lectures at the French summer school of theoretical physics, Cargèse, Corsica, July 1964). 